\title{
Some new inequalities for generalized convex functions pertaining generalized fractional integral operators and their applications
}

\author{
A. KASHURI, M.A. ALI, M. ABBAS AND M. TOSEEF
}

\begin{abstract}
In this paper, authors establish a new identity for a differentiable function using generic integral operators. By applying it, some new integral inequalities of trapezium, Ostrowski and Simpson type are obtained. Moreover, several special cases have been studied in detail. Finally, many useful applications have been found.
\end{abstract}

Mathematics Subject Classification 2010: 26A51; 26A33, 26D07, 26D10, $26 \mathrm{D} 15$.

Keywords: Inequalities; convexity; Raina's function; special means; error estimation.

\section{INTRODUCTION AND PRELIMINARIES}

DEFINITION 1.1. [49] $\Lambda: \mathrm{I} \subseteq \mathbb{R} \rightarrow \mathbb{R}$ is called convex function on $\mathrm{I}$, if

$$
\Lambda\left((1-\zeta) b_{1}+\zeta b_{2}\right) \leq(1-\zeta) \Lambda\left(b_{1}\right)+\zeta \Lambda\left(b_{2}\right), \quad \forall b_{1}, b_{2} \in \mathrm{I}, \zeta \in[0,1]
$$

TheOREM 1.2. (Trapezium inequality) Suppose that $\Lambda: I \subseteq \mathbb{R} \rightarrow \mathbb{R}$ be a convex function, $b_{1}, b_{2} \in \mathrm{I}$ with $b_{1}<b_{2}$, then

$$
\Lambda\left(\frac{b_{1}+b_{2}}{2}\right) \leq \frac{1}{b_{2}-b_{1}} \int_{b_{1}}^{b_{2}} \Lambda(\ell) d \ell \leq \frac{\Lambda\left(b_{1}\right)+\Lambda\left(b_{2}\right)}{2} .
$$

Interested readers are referred to [4]-[6],[15; 19;20;22; 25;26; 28],[33]-[38],[45; 47; $52 ; 53 ; 55 ; 56]$.

TheOREM 1.3. (Ostrowski inequality) Assume that $\Lambda: \mathrm{I} \rightarrow \mathbb{R}$ be a differentiable function on $\mathrm{I}^{\circ}, b_{1}, b_{2} \in \mathrm{I}^{\circ}$ with $b_{1}<b_{2}$. If $\left|\Lambda^{\prime}(\ell)\right| \leq \mathscr{K}$, then

$$
\left|\Lambda(\ell)-\frac{1}{b_{2}-b_{1}} \int_{b_{1}}^{b_{2}} \Lambda(\xi) d \xi\right| \leq \mathscr{K}\left(b_{2}-b_{1}\right)\left[\frac{1}{4}+\frac{\left(\ell-\frac{b_{1}+b_{2}}{2}\right)^{2}}{\left(b_{2}-b_{1}\right)^{2}}\right], \quad \forall \ell \in\left[b_{1}, b_{2}\right] .
$$

For other recent published papers about Ostrowski type inequalities, see [1]-[3],[7][14],[17],[29]-[32],[39]-[41],[43; 44; 50; 54; 57]. 
THEOREM 1.4. (Simpson inequality) Let $\Lambda:\left[b_{1}, b_{2}\right] \rightarrow \mathbb{R}$ be four times differentiable on $\left(b_{1}, b_{2}\right)$ and suppose that

$$
\left\|\Lambda^{(4)}\right\|_{\infty}:=\sup _{\ell \in\left(b_{1}, b_{2}\right)}\left|\Lambda^{(4)}\right|<+\infty .
$$

Then

$$
\left|\int_{b_{1}}^{b_{2}} \Lambda(\ell) d \ell-\frac{b_{2}-b_{1}}{3}\left[\frac{\Lambda\left(b_{1}\right)+\Lambda\left(b_{2}\right)}{2}+2 \Lambda\left(\frac{b_{1}+b_{2}}{2}\right)\right]\right| \leq \frac{\left(b_{2}-b_{1}\right)^{5}}{2880}\left\|\Lambda^{(4)}\right\|_{\infty} .
$$

About Simpson type inequalities, see $[27 ; 42 ; 51 ; 57]$.

In our paper we will establish some new trapezium, Ostrowski and Simpson type inequalities pertaining generalized convex functions with respect to another function. Moreover, many useful applications will be given. Hence, it is important to recall some essential facts relevant to fractional integrals.

DEFINITION 1.5. [34] Assume that $\Lambda \in \mathscr{L}\left[b_{1}, b_{2}\right]$, then $\kappa$-fractional integrals, $\eta, \kappa>0$ with $b_{1} \geq 0$ are

$$
I_{b_{1}^{+}}^{\eta, \kappa} \Lambda\left(\xi_{1}\right)=\frac{1}{\kappa \Gamma_{\kappa}(\eta)} \int_{b_{1}}^{\xi_{1}}\left(\xi_{1}-\xi\right)^{\frac{\eta}{\kappa}-1} \Lambda(\xi) d \xi, \quad b_{1}<\xi_{1},
$$

and

$$
I_{b_{2}^{-}}^{\eta, \kappa} \Lambda\left(\xi_{1}\right)=\frac{1}{\kappa \Gamma_{\kappa}(\eta)} \int_{\xi_{1}}^{b_{2}}\left(\xi-\xi_{1}\right)^{\frac{\eta}{\kappa}-1} \Lambda(\xi) d \xi, \quad b_{2}>\xi_{1}
$$

respectively.

DEFINITION 1.6. [35; 36] $S$ is called $\varpi$-convex set, if

$$
\varpi(\jmath) b_{2}+(1-\varpi(\jmath)) b_{1} \in S, \forall b_{1}, b_{2} \in S, \jmath \in[0,1] .
$$

Definition 1.7. $\Lambda: S \rightarrow \mathbb{R}$ on $\varpi$-convex set $S$ is called $\varpi$-convex function, if

$$
\Lambda\left(b_{1}+\jmath e^{i \omega}\left(b_{2}-b_{1}\right)\right) \leq(1-\jmath) \Lambda\left(b_{1}\right)+\jmath \Lambda\left(b_{2}\right), \forall b_{1}, b_{2} \in S, \jmath \in[0,1] .
$$

Raina, in [48], defined for $\rho, \delta>0$ and $|z|<R$, the following class of functions:

$$
\mathscr{F}_{\rho, \delta}^{\sigma}(z)=\mathscr{F}_{\rho, \delta}^{\sigma(0), \sigma(1), \ldots}(z)=\sum_{k=0}^{+\infty} \frac{\sigma(k)}{\Gamma(\rho k+\delta)} z^{k} .
$$


Choosing $|z| \leq 1$, we take hypergeometric function. For $\sigma=(1,1, \ldots)$ with $\rho=\eta,(\Re(\eta)>0), \delta=1$ and $z \in \mathbb{C}$ in (5), we obtain Mittag-Leffler function

$$
\mathscr{E}_{\eta}(z)=\sum_{k=0}^{+\infty} \frac{z^{k}}{\Gamma(1+\eta k)}
$$

DEFINITION 1.8. $S \neq \emptyset$ is called generalized convex set, if

$$
m b_{1}+\zeta \mathscr{F}_{\rho, \delta}^{\sigma}\left(b_{2}-m b_{1}\right) \in S, \quad \forall b_{1}, b_{2} \in S, \quad m \in(0,1], \quad \zeta \in[0,1] .
$$

DEFINITION 1.9. $\Lambda$ is called generalized convex function, if

$$
\Lambda\left(m b_{1}+\zeta \mathscr{F}_{\rho, \delta}^{\sigma}\left(b_{2}-m b_{1}\right)\right) \leq(1-\zeta) \Lambda\left(m b_{1}\right)+\zeta \Lambda\left(b_{2}\right), \quad \forall b_{1}, b_{2} \in S, \quad m \in(0,1], \quad \zeta \in[0,1] .
$$

REMARK 1.10. Taking $m=1$ and $\mathscr{F}_{\rho, \delta}^{\sigma}\left(b_{2}-b_{1}\right)=b_{2}-b_{1}>0$, we get definition 1.1. For suitable choice of $\mathscr{F}_{\rho, \delta}^{\sigma}(\cdot)$, we obtain definition 1.7. This describes the reasons and motivations of newly defined notions and the relation with these known definitions.

Definition 1.11. [23; 24] Assume that $\Lambda_{2}:\left[b_{1}, b_{2}\right] \rightarrow \mathbb{R}$ is an increasing and positive monotone function on $\left[b_{1}, b_{2}\right]$, with continuous derivative on $\left(b_{1}, b_{2}\right)$. The leftright- fractional integrals of $\Lambda_{1}$ with respect to $\Lambda_{2}$ on $\left[b_{1}, b_{2}\right], \eta>0$ are

$$
I_{b_{1}+}^{\eta, \Lambda_{2}} \Lambda_{1}\left(\xi_{1}\right)=\frac{1}{\Gamma(\eta)} \int_{b_{1}}^{\xi_{1}} \frac{\Lambda_{2}^{\prime}(\xi) \Lambda_{1}(\xi)}{\left[\Lambda_{2}\left(\xi_{1}\right)-\Lambda_{2}(\xi)\right]^{1-\eta}} d \xi, b_{1}<\xi_{1},
$$

and

$$
I_{b_{2}-}^{\eta, \Lambda_{2}} \Lambda_{1}\left(\xi_{1}\right)=\frac{1}{\Gamma(\eta)} \int_{\xi_{1}}^{b_{2}} \frac{\Lambda_{2}^{\prime}(\xi) \Lambda_{1}(\xi)}{\left[\Lambda_{2}(\xi)-\Lambda_{2}\left(\xi_{1}\right)\right]^{1-\eta}} d \xi, b_{2}>\xi_{1},
$$

respectively.

Function $\varpi:[0,+\infty) \rightarrow[0,+\infty)$ constructed from Sarikaya et al. in [45; 46], has the following properties:

$$
\begin{aligned}
& \int_{0}^{1} \frac{\varpi(\xi)}{\xi} d \xi<+\infty \\
& \frac{1}{A_{1}} \leq \frac{\varpi(\varepsilon)}{\varpi\left(\xi_{1}\right)} \leq A_{1} \text { for } \frac{1}{2} \leq \frac{\varepsilon}{\xi_{1}} \leq 2, \\
& \frac{\varpi\left(\xi_{1}\right)}{\xi_{1}^{2}} \leq A_{2} \frac{\varpi(\varepsilon)}{\varepsilon^{2}} \text { for } \varepsilon \leq \xi_{1}, \\
& \left|\frac{\varpi\left(\xi_{1}\right)}{\xi_{1}^{2}}-\frac{\varpi(\varepsilon)}{\varepsilon^{2}}\right| \leq A_{3}\left|\xi_{1}-\varepsilon\right| \frac{\varpi\left(\xi_{1}\right)}{\xi_{1}^{2}} \text { for } \frac{1}{2} \leq \frac{\varepsilon}{\xi_{1}} \leq 2,
\end{aligned}
$$


where $A_{1}, A_{2}, A_{3}>0$ are independent of $\varepsilon, \xi_{1}>0$. Moreover, Sarikaya et al. defined the following useful operators:

$$
\begin{array}{ll}
b_{1}^{+} I_{\varpi} \Lambda\left(\xi_{1}\right)=\int_{b_{1}}^{\xi_{1}} \frac{\varpi\left(\xi_{1}-\xi\right)}{\xi_{1}-\xi} \Lambda(\xi) d \xi, & b_{1}<\xi_{1}, \\
b_{2}^{-} I_{\varpi} \Lambda\left(\xi_{1}\right)=\int_{\xi_{1}}^{b_{2}} \frac{\varpi\left(\xi-\xi_{1}\right)}{\xi-\xi_{1}} \Lambda(\xi) d \xi, & b_{2}>\xi_{1} .
\end{array}
$$

About their efficiency, see [18; 21; 45]. Finally, Farid in [16], defined the following generic operators:

$$
G_{b_{1}+}^{\sigma, \Lambda_{2}} \Lambda_{1}\left(\xi_{1}\right)=\int_{b_{1}}^{\xi_{1}} \frac{\varpi\left(\Lambda_{2}\left(\xi_{1}\right)-\Lambda_{2}(\xi)\right)}{\Lambda_{2}\left(\xi_{1}\right)-\Lambda_{2}(\xi)} \Lambda_{2}^{\prime}(\xi) \Lambda_{1}(\xi) d \xi, b_{1}<\xi_{1},
$$

and

$$
G_{b_{2}-}^{\Phi, \Lambda_{2}} \Lambda_{1}\left(\xi_{1}\right)=\int_{\xi_{1}}^{b_{2}} \frac{\varpi\left(\Lambda_{2}(\xi)-\Lambda_{2}\left(\xi_{1}\right)\right)}{\Lambda_{2}(\xi)-\Lambda_{2}\left(\xi_{1}\right)} \Lambda_{2}^{\prime}(\xi) \Lambda_{1}(\xi) d \xi, b_{2}>\xi_{1},
$$

respectively.

The paper is constructed in this way: In Section 2, we will find an interesting identity with parameter $\lambda$ and using generic integral operators form auxiliary equality, some new integral inequalities of trapezium, Ostrowski and Simpson type will be obtain. Section 3 is devoted to useful applications.

\section{MAIN RESULTS}

Let $\mathscr{P}=\left[m b_{1}, b_{2}\right]$, where $b_{1}<b_{2}$ for some fixed $m \in(0,1]$ with $\zeta \in[0,1]$.

$$
\begin{aligned}
\Pi_{m}^{\varpi, \Upsilon}(\ell, \zeta) & :=\int_{0}^{\zeta} \frac{\varpi\left(\Upsilon\left(m b_{1}+\xi \underset{\rho, \delta}{\sigma}\left(\ell-m b_{1}\right)\right)-\Upsilon\left(m b_{1}\right)\right)}{\Upsilon\left(m b_{1}+\xi \mathscr{F}_{\rho, \delta}^{\sigma}\left(\ell-m b_{1}\right)\right)-\Upsilon\left(m b_{1}\right)} \\
& \times \Upsilon^{\prime}\left(m b_{1}+\xi \mathscr{F}_{\rho, \delta}^{\sigma}\left(\ell-m b_{1}\right)\right) d \xi<+\infty,
\end{aligned}
$$

and

$$
\Xi_{m}^{\sigma, \Upsilon}(\ell, \zeta):=\int_{\zeta}^{1} \frac{\varpi\left(\Upsilon\left(m \ell+\mathscr{F}_{\rho, \delta}^{\sigma}\left(b_{2}-m \ell\right)\right)-\Upsilon\left(m \ell+\xi \underset{\rho, \delta}{\mathscr{F}_{\rho, \delta}^{\sigma}}\left(b_{2}-m \ell\right)\right)\right)}{\Upsilon\left(m \ell+\mathscr{F}_{\rho, \delta}^{\sigma}\left(b_{2}-m \ell\right)\right)-\Upsilon\left(m \ell+\xi \mathscr{F}_{\rho, \delta}^{\sigma}\left(b_{2}-m \ell\right)\right)}
$$




$$
\times \Upsilon^{\prime}\left(m \ell+\xi \mathscr{F}_{\rho, \lambda}^{\sigma}\left(b_{2}-m \ell\right)\right) d \xi<+\infty .
$$

The following lemma will help us to find new results.

LEMma 2.1. Let $\Lambda: \mathscr{P} \rightarrow \mathbb{R}$ be a differentiable function on $\mathscr{P} \circ$ and $\lambda \in \mathbb{R}$. Assume that $\Lambda^{\prime} \in \mathscr{L}(\mathscr{P})$ and $\mathscr{F}_{\rho, \lambda}^{\sigma}\left(b_{2}-m b_{1}\right)>0$, then

$$
\begin{gathered}
\frac{\mathscr{F}_{\rho, \delta}^{\sigma}\left(\ell-m b_{1}\right) \Lambda\left(m b_{1}+\zeta \mathscr{F}_{\rho, \delta}^{\sigma}\left(\ell-m b_{1}\right)\right)+\mathscr{F}_{\rho, \delta}^{\sigma}\left(b_{2}-m \ell\right) \Lambda(m \ell)}{\mathscr{F}_{\rho, \delta}^{\sigma}\left(b_{2}-m b_{1}\right)} \\
-\frac{\lambda}{\mathscr{F}_{\rho, \delta}^{\sigma}\left(b_{2}-m b_{1}\right)} \times\left[\frac{\mathscr{F}_{\rho, \delta}^{\sigma}\left(\ell-m b_{1}\right) \Lambda\left(m b_{1}+\zeta \mathscr{F}_{\rho, \delta}^{\sigma}\left(\ell-m b_{1}\right)\right)}{\prod_{m}^{\sigma, \mathrm{r}}(\ell, 1)}+\frac{\mathscr{F}_{\rho, \delta}^{\sigma}\left(b_{2}-m \ell\right) \Lambda(m \ell)}{\Xi_{m}^{\sigma, \mathrm{r}}(\ell, 0)}\right] \\
+\frac{\lambda}{\mathscr{F}_{\rho, \delta}^{\sigma}\left(b_{2}-m b_{1}\right)} \times\left[\frac{\mathscr{F}_{\rho, \delta}^{\sigma}\left(\ell-m b_{1}\right) \Lambda\left(m b_{1}\right)}{\Pi_{m}^{\sigma, \mathrm{r}}(\ell, 1)}+\frac{\mathscr{F}_{\rho, \delta}^{\sigma}\left(b_{2}-m \ell\right) \Lambda\left(m \ell+\mathscr{F}_{\rho, \delta}^{\sigma}\left(b_{2}-m \ell\right)\right)}{\Xi_{m}^{\sigma, \mathrm{r}}(\ell, 0)}\right] \\
-\frac{1}{\mathscr{F}_{\rho, \delta}^{\sigma}\left(b_{2}-m b_{1}\right)} \times\left[\frac{\left(m b_{1}+\mathscr{F}_{\rho, \delta}^{\sigma}\left(\ell-m b_{1}\right)\right)^{-} \Lambda\left(m b_{1}\right)}{\prod_{m}^{\sigma, \mathrm{r}}(\ell, 1)}+\frac{G_{(m \ell)^{+}}^{\sigma, \mathrm{r}} \Lambda\left(m \ell+\mathscr{F}_{\rho, \delta}^{\sigma}\left(b_{2}-m \ell\right)\right)}{\Xi_{m}^{\sigma, \mathrm{r}}(\ell, 0)}\right] \\
=\frac{\left[\mathscr{F}_{\rho, \delta}^{\sigma}\left(\ell-m b_{1}\right)\right]^{2}}{\prod_{m}^{\sigma, \mathrm{r}}(\ell, 1) \mathscr{F}_{\rho, \delta}^{\sigma}\left(b_{2}-m b_{1}\right)} \times \int_{0}^{1}\left[\Pi_{m}^{\sigma, \mathrm{r}}(\ell, \zeta)-\lambda\right] \Lambda^{\prime}\left(m b_{1}+\zeta \mathscr{F}_{\rho, \delta}^{\sigma}\left(\ell-m b_{1}\right)\right) d \zeta \quad(20) \\
-\frac{\left[\mathscr{F}_{\rho, \delta}^{\sigma}\left(b_{2}-m \ell\right)\right]^{2}}{\Xi_{m}^{\sigma, \mathrm{r}}(\ell, 0) \mathscr{F}_{\rho, \delta}^{\sigma}\left(b_{2}-m b_{1}\right)} \times \int_{0}^{1}\left[\Xi_{m}^{\sigma, \mathrm{r}}(\ell, \zeta)-\lambda\right] \Lambda^{\prime}\left(m \ell+\zeta \mathscr{F}_{\rho, \delta}^{\sigma}\left(b_{2}-m \ell\right)\right) d \zeta .
\end{gathered}
$$

We denote

$$
\begin{gathered}
T_{\Lambda, \Pi_{m}^{\sigma, \mathrm{r}}, \Xi_{m}^{\sigma, \mathrm{r}}}\left(\lambda ; \ell, b_{1}, b_{2}\right):=\frac{\left[\mathscr{F}_{\rho, \delta}^{\sigma}\left(\ell-m b_{1}\right)\right]^{2}}{\Pi_{m}^{\sigma, \mathrm{r}}(\ell, 1) \mathscr{F}_{\rho, \delta}^{\sigma}\left(b_{2}-m b_{1}\right)} \\
\times \int_{0}^{1}\left[\Pi_{m}^{\sigma, \mathrm{r}}(\ell, \zeta)-\lambda\right] \Lambda^{\prime}\left(m b_{1}+\zeta \mathscr{F}_{\rho, \delta}^{\sigma}\left(\ell-m b_{1}\right)\right) d \zeta \\
-\frac{\left[\mathscr{F}_{\rho, \delta}^{\sigma}\left(b_{2}-m \ell\right)\right]^{2}}{\Xi_{m}^{\sigma, \mathrm{r}}(\ell, 0) \mathscr{F}_{\rho, \delta}^{\sigma}\left(b_{2}-m b_{1}\right)} \times \int_{0}^{1}\left[\Xi_{m}^{\sigma, \mathrm{r}}(\ell, \zeta)-\lambda\right] \Lambda^{\prime}\left(m \ell+\zeta \mathscr{F}_{\rho, \delta}^{\sigma}\left(b_{2}-m \ell\right)\right) d \zeta .
\end{gathered}
$$


PROOF. By integrating by parts (21), we derive

$$
\begin{aligned}
& T_{\Lambda, \Pi_{m}^{\Phi, \mathrm{r}}, \mathbb{\Xi}_{m}^{\Phi, \mathrm{r}}}\left(\lambda ; \ell, b_{1}, b_{2}\right)=\frac{\left[\mathscr{F}_{\rho, \delta}^{\sigma}\left(\ell-m b_{1}\right)\right]^{2}}{\Pi_{m}^{\sigma, \mathrm{r}}(\ell, 1) \mathscr{F}_{\rho, \delta}^{\sigma}\left(b_{2}-m b_{1}\right)} \\
& \times\left\{\int_{0}^{1} \Pi_{m}^{\sigma, \Upsilon}(\ell, \zeta) \Lambda^{\prime}\left(m b_{1}+\zeta \mathscr{F}_{\rho, \delta}^{\sigma}\left(\ell-m b_{1}\right)\right) d \zeta-\lambda \int_{0}^{1} \Lambda^{\prime}\left(m b_{1}+\zeta \mathscr{F}_{\rho, \delta}^{\sigma}\left(\ell-m b_{1}\right)\right) d \zeta\right\} \\
& -\frac{\left[\mathscr{F}_{\rho, \delta}^{\sigma}\left(b_{2}-m \ell\right)\right]^{2}}{\Xi_{m}^{\sigma, \Upsilon}(\ell, 0) \mathscr{F}_{\rho, \delta}^{\sigma}\left(b_{2}-m b_{1}\right)} \\
& \times\left\{\int_{0}^{1} \Xi_{m}^{\sigma, \mathrm{r}}(\ell, \zeta) \Lambda^{\prime}\left(m \ell+\zeta \mathscr{F}_{\rho, \delta}^{\sigma}\left(b_{2}-m \ell\right)\right) d \zeta-\lambda \int_{0}^{1} \Lambda^{\prime}\left(m \ell+\zeta \mathscr{F}_{\rho, \delta}^{\sigma}\left(b_{2}-m \ell\right)\right) d \zeta\right\} \\
& =\frac{\left[\mathscr{F}_{\rho, \delta}^{\sigma}\left(\ell-m b_{1}\right)\right]^{2}}{\prod_{m}^{\Phi, \mathrm{\Upsilon}}(\ell, 1) \mathscr{F}_{\rho, \delta}^{\sigma}\left(b_{2}-m b_{1}\right)} \times\left\{\left.\frac{\Pi_{m}^{\sigma, \Upsilon}(\ell, \zeta) \Lambda\left(m b_{1}+\zeta \mathscr{F}_{\rho, \delta}^{\sigma}\left(\ell-m b_{1}\right)\right)}{\mathscr{F}_{\rho, \delta}^{\sigma}\left(\ell-m b_{1}\right)}\right|_{0} ^{1}-\frac{1}{\mathscr{F}_{\rho, \delta}^{\sigma}\left(\ell-m b_{1}\right)}\right. \\
& \times \int_{0}^{1} \frac{\varpi\left(\Upsilon\left(m b_{1}+\zeta \mathscr{F}_{\rho, \delta}^{\sigma}\left(\ell-m b_{1}\right)\right)-\Upsilon\left(m b_{1}\right)\right)}{\Upsilon\left(m b_{1}+\zeta \mathscr{F}_{\rho, \delta}^{\sigma}\left(\ell-m b_{1}\right)\right)-\Upsilon\left(m b_{1}\right)} \\
& \times \Upsilon^{\prime}\left(m b_{1}+\zeta \mathscr{F}_{\rho, \delta}^{\sigma}\left(\ell-m b_{1}\right)\right) \Lambda\left(m b_{1}+\zeta \mathscr{F}_{\rho, \delta}^{\sigma}\left(\ell-m b_{1}\right)\right) d \zeta \\
& \left.-\left.\frac{\lambda}{\mathscr{F}_{\rho, \delta}^{\sigma}\left(\ell-m b_{1}\right)} \Lambda\left(m b_{1}+\zeta \mathscr{F}_{\rho, \delta}^{\sigma}\left(\ell-m b_{1}\right)\right)\right|_{0} ^{1}\right\}-\frac{\left[\mathscr{F}_{\rho, \delta}^{\sigma}\left(b_{2}-m \ell\right)\right]^{2}}{\Xi_{m}^{\sigma, Y}(\ell, 0) \mathscr{F}_{\rho, \delta}^{\sigma}\left(b_{2}-m b_{1}\right)} \\
& \times\left\{\left.\frac{\Xi_{m}^{\sigma, \mathrm{r}}(\ell, \zeta) \Lambda\left(m \ell+\zeta \mathscr{F}_{\rho, \delta}^{\sigma}\left(b_{2}-m \ell\right)\right)}{\mathscr{F}_{\rho, \delta}^{\sigma}\left(b_{2}-m \ell\right)}\right|_{0} ^{1}\right. \\
& -\frac{1}{\mathscr{F}_{\rho, \delta}^{\sigma}\left(b_{2}-m \ell\right)} \times \int_{0}^{1} \frac{\varpi\left(\Upsilon\left(m \ell+\mathscr{F}_{\rho, \delta}^{\sigma}\left(b_{2}-m \ell\right)\right)-\Upsilon\left(m \ell+\zeta \mathscr{F}_{\rho, \delta}^{\sigma}\left(b_{2}-m \ell\right)\right)\right)}{\Upsilon\left(m \ell+\mathscr{F}_{\rho, \delta}^{\sigma}\left(b_{2}-m \ell\right)\right)-\Upsilon\left(m \ell+\zeta_{\rho, \delta}^{\sigma}\left(b_{2}-m \ell\right)\right)} \\
& \times \Upsilon^{\prime}\left(m \ell+\zeta \mathscr{F}_{\rho, \delta}^{\sigma}\left(b_{2}-m \ell\right)\right) \Lambda\left(m \ell+\zeta \mathscr{F}_{\rho, \delta}^{\sigma}\left(b_{2}-m \ell\right)\right) d \zeta \\
& \left.-\left.\frac{\lambda}{\mathscr{F}_{\rho, \delta}^{\sigma}\left(b_{2}-m \ell\right)} \Lambda\left(m \ell+\zeta_{\rho, \delta}^{\sigma}\left(b_{2}-m \ell\right)\right)\right|_{0} ^{1}\right\}
\end{aligned}
$$




$$
\begin{aligned}
& =\frac{\mathscr{F}_{\rho, \delta}^{\sigma}\left(\ell-m b_{1}\right) \Lambda\left(m b_{1}+\zeta \mathscr{F}_{\rho, \delta}^{\sigma}\left(\ell-m b_{1}\right)\right)+\mathscr{F}_{\rho, \delta}^{\sigma}\left(b_{2}-m \ell\right) \Lambda(m \ell)}{\mathscr{F}_{\rho, \delta}^{\sigma}\left(b_{2}-m b_{1}\right)} \\
& -\frac{\lambda}{\mathscr{F}_{\rho, \delta}^{\sigma}\left(b_{2}-m b_{1}\right)} \times\left[\frac{\mathscr{F}_{\rho, \delta}^{\sigma}\left(\ell-m b_{1}\right) \Lambda\left(m b_{1}+\zeta \mathscr{F}_{\rho, \delta}^{\sigma}\left(\ell-m b_{1}\right)\right)}{\prod_{m}^{\sigma, \mathrm{r}}(\ell, 1)}+\frac{\mathscr{F}_{\rho, \delta}^{\sigma}\left(b_{2}-m \ell\right) \Lambda(m \ell)}{\Xi_{m}^{\sigma, \mathrm{r}}(\ell, 0)}\right] \\
& +\frac{\lambda}{\mathscr{F}_{\rho, \delta}^{\sigma}\left(b_{2}-m b_{1}\right)} \times\left[\frac{\mathscr{F}_{\rho, \delta}^{\sigma}\left(\ell-m b_{1}\right) \Lambda\left(m b_{1}\right)}{\prod_{m}^{\sigma, \mathrm{r}}(\ell, 1)}+\frac{\mathscr{F}_{\rho, \delta}^{\sigma}\left(b_{2}-m \ell\right) \Lambda\left(m \ell+\mathscr{F}_{\rho, \delta}^{\sigma}\left(b_{2}-m \ell\right)\right)}{\Xi_{m}^{\sigma, \mathrm{r}}(\ell, 0)}\right] \\
& -\frac{1}{\mathscr{F}_{\rho, \delta}^{\sigma}\left(b_{2}-m b_{1}\right)} \times\left[\frac{G_{\left(m b_{1}+\mathscr{F}_{\rho, \delta}^{\sigma}\left(\ell-m b_{1}\right)\right)^{-}}^{\sigma, \Gamma}\left(m b_{1}\right)}{\prod_{m}^{\sigma,,}(\ell, 1)}+\frac{G_{(m \ell)^{+}}^{\sigma, \Upsilon} \Lambda\left(m \ell+\mathscr{F}_{\rho, \delta}^{\sigma}\left(b_{2}-m \ell\right)\right)}{\Xi_{m}^{\sigma, \Gamma}(\ell, 0)}\right] .
\end{aligned}
$$

REMARK 2.2. a. Taking $m=1, \lambda=0, \mathscr{F}_{\rho, \delta}^{\sigma}\left(\ell-m b_{1}\right)=\ell-m b_{1}, \mathscr{F}_{\rho, \delta}^{\sigma}\left(b_{2}-m \ell\right)=$ $b_{2}-m \ell, \mathscr{F}_{\rho, \delta}^{\sigma}\left(b_{2}-m b_{1}\right)=b_{2}-m b_{1}$ and $\Upsilon(\zeta)=\zeta=\varpi(\zeta)$ in Lemma 2.1, then

$$
T_{\Lambda}\left(\ell, b_{1}, b_{2}\right):=\Lambda(\ell)-\frac{1}{b_{2}-b_{1}} \int_{b_{1}}^{b_{2}} \Lambda(\zeta) d \zeta
$$

b. Choosing $m=1, \lambda=1, \mathscr{F}_{\rho, \delta}^{\sigma}\left(\ell-m b_{1}\right)=\ell-m b_{1}, \mathscr{F}_{\rho, \delta}^{\sigma}\left(b_{2}-m \ell\right)=b_{2}-m \ell, \mathscr{F}_{\rho, \delta}^{\sigma}\left(b_{2}-\right.$ $\left.m b_{1}\right)=b_{2}-m b_{1}$ and $\Upsilon(\zeta)=\zeta=\varpi(\zeta)$ in Lemma 2.1 , then

$$
\overline{T_{\Lambda}}\left(\ell, b_{1}, b_{2}\right):=\frac{\left(\ell-b_{1}\right) \Lambda\left(b_{1}\right)+\left(b_{2}-\ell\right) \Lambda\left(b_{2}\right)}{b_{2}-b_{1}}-\frac{1}{b_{2}-b_{1}} \int_{b_{1}}^{b_{2}} \Lambda(\zeta) d \zeta
$$

c. Taking $m=1, \ell=\frac{b_{1}+b_{2}}{2}, \mathscr{F}_{\rho, \delta}^{\sigma}\left(\ell-m b_{1}\right)=\ell-m b_{1}, \mathscr{F}_{\rho, \delta}^{\sigma}\left(b_{2}-m \ell\right)=b_{2}-m \ell, \mathscr{F}_{\rho, \delta}^{\sigma}\left(b_{2}-\right.$ $\left.m b_{1}\right)=b_{2}-m b_{1}$ and $\Upsilon(\zeta)=\zeta=\varpi(\zeta)$ in Lemma 2.1 , then

$$
T_{\Lambda}\left(\lambda ; b_{1}, b_{2}\right):=\lambda\left[\frac{\Lambda\left(b_{1}\right)+\Lambda\left(b_{2}\right)}{2}\right]+(1-\lambda) \Lambda\left(\frac{b_{1}+b_{2}}{2}\right)-\frac{1}{b_{2}-b_{1}} \int_{b_{1}}^{b_{2}} \Lambda(\zeta) d \zeta .
$$

TheOREM 2.3. Let $\Lambda: \mathscr{P} \rightarrow \mathbb{R}$ be a differentiable function on $\mathscr{P}{ }^{\circ}$ and $\lambda \in[0,1]$. If $\left|\Lambda^{\prime}\right|^{q}$ is generalized convex function on $\mathscr{P}$ and $\mathscr{F}_{\rho, \delta}^{\sigma}\left(b_{2}-m b_{1}\right)>0$, then for $q>1$ and $\frac{1}{p}+\frac{1}{q}=1$, we have

$$
\begin{gathered}
\left|T_{\Lambda, \Pi_{m}^{\sigma, \Gamma}, \mathbb{\Xi}_{m}^{\sigma, \mathrm{r}}}\left(\lambda ; \ell, b_{1}, b_{2}\right)\right| \\
\leq \frac{\left[\mathscr{F}_{\rho, \delta}^{\sigma}\left(\ell-m b_{1}\right)\right]^{2}}{\sqrt[q]{2} \Pi_{m}^{\sigma, \mathrm{r}}(\ell, 1) \mathscr{F}_{\rho, \delta}^{\sigma}\left(b_{2}-m b_{1}\right)} \sqrt[p]{B_{\Pi_{m}}^{\sigma, \mathrm{r}}(\ell ; \lambda, p)} \times \sqrt[q]{\left|\Lambda^{\prime}\left(m b_{1}\right)\right|^{q}+\left|\Lambda^{\prime}(\ell)\right|^{q}}
\end{gathered}
$$




$$
+\frac{\left[\mathscr{F}_{\rho, \delta}^{\sigma}\left(b_{2}-m \ell\right)\right]^{2}}{\sqrt[q]{2} \Xi_{m}^{\sigma, \Upsilon}(\ell, 0) \mathscr{F}_{\rho, \delta}^{\sigma}\left(b_{2}-m b_{1}\right)} \sqrt[p]{B_{\Xi_{m}}^{\sigma, \Upsilon}(\ell ; \lambda, p)} \times \sqrt[q]{\left|\Lambda^{\prime}(m \ell)\right|^{q}+\left|\Lambda^{\prime}\left(b_{2}\right)\right|^{q}},
$$

where

$$
B_{\Pi_{m}}^{\sigma, \mathrm{r}}(\ell ; \lambda, p):=\int_{0}^{1}\left|\Pi_{m}^{\sigma, \mathrm{r}}(\ell, \zeta)-\lambda\right|^{p} d \zeta, \quad B_{\Xi_{m}}^{\varpi, \mathrm{r}}(\ell ; \lambda, p):=\int_{0}^{1}\left|\Xi_{m}^{\sigma, \mathrm{r}}(\ell, \zeta)-\lambda\right|^{p} d \zeta
$$

ProOF. Applying Lemma 2.1, generalized convexity of $\left|\Lambda^{\prime}\right|^{q}$, Hölder's inequality, we get

$$
\begin{aligned}
& \left|T_{\Lambda, \Pi_{m}^{\sigma, \mathrm{r}}, \mathbb{\Xi}_{m}^{\bar{G}, \mathrm{\Upsilon}}}\left(\lambda ; \ell, b_{1}, b_{2}\right)\right| \\
& \leq \frac{\left[\mathscr{F}_{\rho, \delta}^{\sigma}\left(\ell-m b_{1}\right)\right]^{2}}{\Pi_{m}^{\sigma, \Upsilon}(\ell, 1) \mathscr{F}_{\rho, \delta}^{\sigma}\left(b_{2}-m b_{1}\right)} \times \int_{0}^{1}\left|\Pi_{m}^{\sigma, \Upsilon}(\ell, \zeta)-\lambda\right|\left|\Lambda^{\prime}\left(m b_{1}+\zeta \mathscr{F}_{\rho, \delta}^{\sigma}\left(\ell-m b_{1}\right)\right)\right| d \zeta \\
& +\frac{\left[\mathscr{F}_{\rho, \delta}^{\sigma}\left(b_{2}-m \ell\right)\right]^{2}}{\Xi_{m}^{\sigma, \Upsilon}(\ell, 0) \mathscr{F}_{\rho, \delta}^{\sigma}\left(b_{2}-m b_{1}\right)} \times \int_{0}^{1}\left|\Xi_{m}^{\sigma, \Upsilon}(\ell, \zeta)-\lambda\right|\left|\Lambda^{\prime}\left(m \ell+\zeta_{\rho, \delta}^{\sigma}\left(b_{2}-m \ell\right)\right)\right| d \zeta \\
& \leq \frac{\left[\mathscr{F}_{\rho, \delta}^{\sigma}\left(\ell-m b_{1}\right)\right]^{2}}{\prod_{m}^{\bar{\sigma}, \mathrm{r}}(\ell, 1) \mathscr{F}_{\rho, \delta}^{\sigma}\left(b_{2}-m b_{1}\right)} \\
& \times\left(\int_{0}^{1}\left|\Pi_{m}^{\Phi, r}(\ell, \zeta)-\lambda\right|^{p} d \zeta\right)^{\frac{1}{p}}\left(\int_{0}^{1}\left|\Lambda^{\prime}\left(m b_{1}+\zeta \mathscr{F}_{\rho, \delta}^{\sigma}\left(\ell-m b_{1}\right)\right)\right|^{q} d \zeta\right)^{\frac{1}{q}} \\
& +\frac{\left[\mathscr{F}_{\rho, \delta}^{\sigma}\left(b_{2}-m \ell\right)\right]^{2}}{\Xi_{m}^{\sigma, \Upsilon}(\ell, 0) \mathscr{F}_{\rho, \delta}^{\sigma}\left(b_{2}-m b_{1}\right)} \\
& \times\left(\int_{0}^{1}\left|\Xi_{m}^{\sigma, \mathrm{r}}(\ell, \zeta)-\lambda\right|^{p} d \zeta\right)^{\frac{1}{p}}\left(\int_{0}^{1}\left|\Lambda^{\prime}\left(m \ell+\zeta \mathscr{F}_{\rho, \delta}^{\sigma}\left(b_{2}-m \ell\right)\right)\right|^{q} d \zeta\right)^{\frac{1}{q}} \\
& \leq \frac{\left[\mathscr{F}_{\rho, \delta}^{\sigma}\left(\ell-m b_{1}\right)\right]^{2}}{\prod_{m}^{\sigma, \mathrm{r}}(\ell, 1) \mathscr{F}_{\rho, \delta}^{\sigma}\left(b_{2}-m b_{1}\right)} \sqrt[p]{B_{\Pi_{m}}^{\sigma, \mathrm{r}}(\ell ; \lambda, p)}\left(\int_{0}^{1}\left[(1-\zeta)\left|\Lambda^{\prime}\left(m b_{1}\right)\right|^{q}+\zeta\left|\Lambda^{\prime}(\ell)\right|^{q}\right] d \zeta\right)^{\frac{1}{q}} \\
& +\frac{\left[\mathscr{F}_{\rho, \delta}^{\sigma}\left(b_{2}-m \ell\right)\right]^{2}}{\Xi_{m}^{\sigma, \Gamma}(\ell, 0) \mathscr{F}_{\rho, \delta}^{\sigma}\left(b_{2}-m b_{1}\right)} \sqrt[p]{B_{\Xi_{m}}^{\sigma, \Upsilon}(\ell ; \lambda, p)}\left(\int_{0}^{1}\left[(1-\zeta)\left|\Lambda^{\prime}(m \ell)\right|^{q}+\zeta\left|\Lambda^{\prime}\left(b_{2}\right)\right|^{q}\right] d \zeta\right)^{\frac{1}{q}}
\end{aligned}
$$




$$
\begin{gathered}
=\frac{\left[\mathscr{F}_{\rho, \delta}^{\sigma}\left(\ell-m b_{1}\right)\right]^{2}}{\sqrt[q]{2} \Pi_{m}^{\sigma, \mathrm{r}}(\ell, 1) \mathscr{F}_{\rho, \delta}^{\sigma}\left(b_{2}-m b_{1}\right)} \sqrt[p]{B_{\Pi_{m}}^{\sigma, \mathrm{r}}(\ell ; \lambda, p)} \times \sqrt[q]{\left|\Lambda^{\prime}\left(m b_{1}\right)\right|^{q}+\left|\Lambda^{\prime}(\ell)\right|^{q}} \\
+\frac{\left[\mathscr{F}_{\rho, \delta}^{\sigma}\left(b_{2}-m \ell\right)\right]^{2}}{\sqrt[q]{2} \Xi_{m}^{\sigma, \mathrm{r}}(\ell, 0) \mathscr{F}_{\rho, \delta}^{\sigma}\left(b_{2}-m b_{1}\right)} \sqrt[p]{B_{\Xi_{m}}^{\sigma, \mathrm{r}}(\ell ; \lambda, p)} \times \sqrt[q]{\left|\Lambda^{\prime}(m \ell)\right|^{q}+\left|\Lambda^{\prime}\left(b_{2}\right)\right|^{q}}
\end{gathered}
$$

COROLlary 2.4. Taking $p=2=q$ in Theorem 2.3, we have

$$
\begin{gathered}
\left|T_{\Lambda, \Pi_{m}^{\sigma, \mathrm{r}}, \mathbb{\Xi}_{m}^{\sigma, \mathrm{r}}}\left(\lambda ; \ell, b_{1}, b_{2}\right)\right| \\
\leq \frac{\left[\mathscr{F}_{\rho, \delta}^{\sigma}\left(\ell-m b_{1}\right)\right]^{2}}{\sqrt{2} \Pi_{m}^{\sigma, \mathrm{r}}(\ell, 1) \mathscr{F}_{\rho, \delta}^{\sigma}\left(b_{2}-m b_{1}\right)} \sqrt{B_{\Pi_{m}}^{\sigma, \mathrm{r}}(\ell ; \lambda, 2)} \times \sqrt{\left|\Lambda^{\prime}\left(m b_{1}\right)\right|^{2}+\left|\Lambda^{\prime}(\ell)\right|^{2}} \\
+\frac{\left[\mathscr{F}_{\rho, \delta}^{\sigma}\left(b_{2}-m \ell\right)\right]^{2}}{\sqrt{2} \Xi_{m}^{\sigma, \mathrm{r}}(\ell, 0) \mathscr{F}_{\rho, \delta}^{\sigma}\left(b_{2}-m b_{1}\right)} \sqrt{B_{\Xi_{m}}^{\sigma, \mathrm{r}}(\ell ; \lambda, 2)} \times \sqrt{\left|\Lambda^{\prime}(m \ell)\right|^{2}+\left|\Lambda^{\prime}\left(b_{2}\right)\right|^{2}} .
\end{gathered}
$$

COROLlary 2.5. Choosing $\left|\Lambda^{\prime}\right| \leq \mathscr{K}$ in Theorem 2.3, we get

$$
\begin{gathered}
\left|T_{\Lambda, \Pi_{m}^{\Pi, \mathrm{r}}, \Xi_{m}^{\sigma, \mathrm{r}}}\left(\lambda ; \ell, b_{1}, b_{2}\right)\right| \leq \frac{\mathscr{K}}{\mathscr{F}_{\rho, \delta}^{\sigma}\left(b_{2}-m b_{1}\right)} \\
\times\left[\frac{\left[\mathscr{F}_{\rho, \delta}^{\sigma}\left(\ell-m b_{1}\right)\right]^{2}}{\Pi_{m}^{\sigma, \mathrm{r}}(\ell, 1)} \sqrt[p]{B_{\Pi_{m}}^{\sigma, \mathrm{r}}(\ell ; \lambda, p)}+\frac{\left[\mathscr{F}_{\rho, \delta}^{\sigma}\left(b_{2}-m \ell\right)\right]^{2}}{\Xi_{m}^{\sigma, \mathrm{r}}(\ell, 0)} \sqrt[p]{B_{\Xi_{m}}^{\sigma, \mathrm{r}}(\ell ; \lambda, p)}\right] .
\end{gathered}
$$

COROLlaRY 2.6. Taking $m=1, \lambda=0, \mathscr{F}_{\rho, \delta}^{\sigma}\left(\ell-m b_{1}\right)=\ell-m b_{1}, \mathscr{F}_{\rho, \delta}^{\sigma}\left(b_{2}-m \ell\right)=$ $b_{2}-m \ell, \mathscr{F}_{\rho, \delta}^{\sigma}\left(b_{2}-m b_{1}\right)=b_{2}-m b_{1}$ and $\Upsilon(\zeta)=\zeta=\varpi(\zeta)$ in Theorem 2.3, we obtain

$$
\begin{gathered}
\left|T_{\Lambda}\left(\ell, b_{1}, b_{2}\right)\right| \leq \frac{1}{\sqrt[q]{2} \sqrt[p]{p+1}\left(b_{2}-b_{1}\right)} \\
\times\left\{\left(\ell-b_{1}\right)^{2} \sqrt[q]{\left|\Lambda^{\prime}\left(b_{1}\right)\right|^{q}+\left|\Lambda^{\prime}(\ell)\right|^{q}}+\left(b_{2}-\ell\right)^{2} \sqrt[q]{\left.\left|\Lambda^{\prime}(\ell)\right|\right|^{q}+\left|\Lambda^{\prime}\left(b_{2}\right)\right|^{q}}\right\} .
\end{gathered}
$$

Corollary 2.7. Choosing $\ell=\frac{b_{1}+b_{2}}{2}$ in Corollary 2.6, we have

$$
\begin{gathered}
\left|T_{\Lambda}\left(b_{1}, b_{2}\right)\right| \leq \frac{\left(b_{2}-b_{1}\right)}{4 \sqrt[q]{2} \sqrt[p]{p+1}} \\
\times\left\{\sqrt[q]{\left|\Lambda^{\prime}\left(b_{1}\right)\right|^{q}+\left|\Lambda^{\prime}\left(\frac{b_{1}+b_{2}}{2}\right)\right|^{q}}+\sqrt[q]{\left|\Lambda^{\prime}\left(\frac{b_{1}+b_{2}}{2}\right)\right|^{q}+\left|\Lambda^{\prime}\left(b_{2}\right)\right|^{q}}\right\} .
\end{gathered}
$$

COROLLARY 2.8. Taking $m=1, \lambda=1, \mathscr{F}_{\rho, \delta}^{\sigma}\left(\ell-m b_{1}\right)=\ell-m b_{1}, \mathscr{F}_{\rho, \delta}^{\sigma}\left(b_{2}-m \ell\right)=$ 
$b_{2}-m \ell, \underset{\rho, \delta}{\sigma}\left(b_{2}-m b_{1}\right)=b_{2}-m b_{1}$ and $\Upsilon(\zeta)=\zeta=\varpi(\zeta)$ in Theorem 2.3, we get

$$
\begin{gathered}
\left|\overline{T_{\Lambda}}\left(\ell, b_{1}, b_{2}\right)\right| \leq \frac{1}{\sqrt[q]{2} \sqrt[p]{p+1}\left(b_{2}-b_{1}\right)} \\
\times\left\{\left(\ell-b_{1}\right)^{2} \sqrt[q]{\left|\Lambda^{\prime}\left(b_{1}\right)\right|^{q}+\left|\Lambda^{\prime}(\ell)\right|^{q}}+\left(b_{2}-\ell\right)^{2} \sqrt[q]{\left.\left|\Lambda^{\prime}(\ell)\right|\right|^{q}+\left|\Lambda^{\prime}\left(b_{2}\right)\right|^{q}}\right\} .
\end{gathered}
$$

COROLlaRY 2.9. Choosing $m=1, \lambda=\frac{1}{3}, \underset{F_{\rho, \delta}}{\sigma}\left(\ell-m b_{1}\right)=\ell-m b_{1}, \mathscr{F}_{\rho, \delta}^{\sigma}\left(b_{2}-\right.$ $m \ell)=b_{2}-m \ell, \mathscr{F}_{\rho, \delta}^{\sigma}\left(b_{2}-m b_{1}\right)=b_{2}-m b_{1}$ and $\Upsilon(\zeta)=\zeta=\varpi(\zeta)$ in Theorem 2.3, we obtain

$$
\begin{gathered}
\left|T_{\Lambda}\left(\frac{1}{3} ; b_{1}, b_{2}\right)\right| \leq \frac{1}{\sqrt[q]{2}\left(b_{2}-b_{1}\right)} \sqrt[p]{\frac{2^{p+1}+1}{3^{p+1}(p+1)}} \\
\times\left\{\left(\ell-b_{1}\right)^{2} \sqrt[q]{\left|\Lambda^{\prime}\left(b_{1}\right)\right|^{q}+\left|\Lambda^{\prime}(\ell)\right|^{q}}+\left(b_{2}-\ell\right)^{2} \sqrt[q]{\left|\Lambda^{\prime}(\ell)\right|^{q}+\left|\Lambda^{\prime}\left(b_{2}\right)\right|^{q}}\right\} .
\end{gathered}
$$

COROLlary 2.10. Substituting $\lambda=0$ and $\varpi(\zeta)=\zeta$ in Theorem 2.3, we have

$$
\begin{gathered}
\left|T_{\Lambda, \Pi_{m}^{r}, \Xi_{m}^{r}}\left(0 ; \ell, b_{1}, b_{2}\right)\right| \leq \frac{1}{\sqrt[q]{2} \mathscr{F}_{\rho, \delta}^{\sigma}\left(b_{2}-m b_{1}\right)} \\
\times\left\{\sqrt[q]{\mathscr{F}_{\rho, \delta}^{\sigma}\left(\ell-m b_{1}\right)} \sqrt[p]{B_{1}^{\Upsilon}(\ell ; p)} \times \sqrt[q]{\left|\Lambda^{\prime}\left(m b_{1}\right)\right|^{q}+\left|\Lambda^{\prime}(\ell)\right|^{q}}\right. \\
\left.+\sqrt[q]{\mathscr{F}_{\rho, \delta}^{\sigma}\left(b_{2}-m \ell\right)} \sqrt[p]{B_{2}^{\Upsilon}(\ell ; p)} \times \sqrt[q]{\left|\Lambda^{\prime}(m \ell)\right|^{q}+\left|\Lambda^{\prime}\left(b_{2}\right)\right|^{q}}\right\},
\end{gathered}
$$

where

$$
B_{1}^{\Upsilon}(\ell ; p):=\int_{m b_{1}}^{m b_{1}+\mathscr{F}_{\rho, \delta}^{\sigma}\left(\ell-m b_{1}\right)}\left[\Upsilon(\zeta)-\Upsilon\left(m b_{1}\right)\right]^{p} d \zeta
$$

and

$$
B_{2}^{\Upsilon}(\ell ; p):=\int_{m \ell}^{m \ell+\mathscr{F}_{\rho, \delta}^{\sigma}\left(b_{2}-m \ell\right)}\left[\Upsilon\left(m \ell+\mathscr{F}_{\rho, \delta}^{\sigma}\left(b_{2}-m \ell\right)\right)-\Upsilon(\zeta)\right]^{p} d \zeta .
$$

COROLlary 2.11. For $\lambda=0$ and $\varpi(\zeta)=\frac{\zeta^{\alpha}}{\Gamma(\alpha)}$ in Theorem 2.3, we get

$$
\left|T_{\Lambda, \Pi_{m}, \Xi_{m}^{r}}\left(0 ; \ell, b_{1}, b_{2}\right)\right| \leq \frac{1}{\sqrt[q]{2} \mathscr{F}_{\rho, \delta}^{\sigma}\left(b_{2}-m b_{1}\right)}
$$




$$
\begin{aligned}
& \times\left\{\sqrt[q]{\mathscr{F}_{\rho, \delta}^{\sigma}\left(\ell-m b_{1}\right)} \sqrt[p]{B_{3}^{\Upsilon}(\ell ; p, \alpha)} \times \sqrt[q]{\left|\Lambda^{\prime}\left(m b_{1}\right)\right|^{q}+\left|\Lambda^{\prime}(\ell)\right|^{q}}\right. \\
& \left.+\sqrt[q]{\mathscr{F}_{\rho, \delta}^{\sigma}\left(b_{2}-m \ell\right)} \sqrt[p]{B_{4}^{\Upsilon}(\ell ; p, \alpha)} \times \sqrt[q]{\left|\Lambda^{\prime}(m \ell)\right|^{q}+\left|\Lambda^{\prime}\left(b_{2}\right)\right|^{q}}\right\},
\end{aligned}
$$

where

$$
B_{3}^{\Upsilon}(\ell ; p, \alpha):=\int_{m b_{1}}^{m b_{1}+\mathscr{F}_{\rho, \delta}^{\sigma}\left(\ell-m b_{1}\right)}\left[\Upsilon(\zeta)-\Upsilon\left(m b_{1}\right)\right]^{p \alpha} d \zeta
$$

and

$$
B_{4}^{\Upsilon}(\ell ; p, \alpha):=\int_{m \ell}^{m \ell+\mathscr{F}_{\rho, \delta}^{\sigma}\left(b_{2}-m \ell\right)}\left[\Upsilon\left(m \ell+\mathscr{F}_{\rho, \delta}^{\sigma}\left(b_{2}-m \ell\right)\right)-\Upsilon(\zeta)\right]^{p \alpha} d \zeta
$$

Corollary 2.12. Substituting $\lambda=0$ and $\varpi(\zeta)=\frac{\zeta^{\alpha}}{\kappa \Gamma_{\kappa}(\alpha)}$ in Theorem 2.3, we obtain

$$
\begin{gathered}
\left|T_{\Lambda, \Pi_{m}, \Xi_{m}^{\Upsilon}}\left(0 ; \ell, b_{1}, b_{2}\right)\right| \leq \frac{1}{\sqrt[q]{2} \mathscr{F}_{\rho, \delta}^{\sigma}\left(b_{2}-m b_{1}\right)} \\
\times\left\{\sqrt[q]{\mathscr{F}_{\rho, \delta}^{\sigma}\left(\ell-m b_{1}\right)} \sqrt[p]{B_{5}^{\Upsilon}(\ell ; p, \alpha, \kappa)} \times \sqrt[q]{\left|\Lambda^{\prime}\left(m b_{1}\right)\right|^{q}+\left|\Lambda^{\prime}(\ell)\right|^{q}}\right. \\
\left.+\sqrt[q]{\mathscr{F}_{\rho, \delta}^{\sigma}\left(b_{2}-m \ell\right)} \sqrt[p]{B_{6}^{\Upsilon}(\ell ; p, \alpha, \kappa)} \times \sqrt[q]{\left|\Lambda^{\prime}(m \ell)\right|^{q}+\left|\Lambda^{\prime}\left(b_{2}\right)\right|^{q}}\right\},
\end{gathered}
$$

where

$$
B_{5}^{\Upsilon}(\ell ; p, \alpha, \kappa):=\int_{m b_{1}}^{m b_{1}+\mathscr{F}_{\rho, \delta}^{\sigma}\left(\ell-m b_{1}\right)}\left[\Upsilon(\zeta)-\Upsilon\left(m b_{1}\right)\right]^{\frac{p \alpha}{\kappa}} d \zeta
$$

and

$$
B_{6}^{\Upsilon}(\ell ; p, \alpha, \kappa):=\int_{m \ell}^{m \ell+\mathscr{F}_{\rho, \delta}^{\sigma}\left(b_{2}-m \ell\right)}\left[\Upsilon\left(m \ell+\mathscr{F}_{\rho, \delta}^{\sigma}\left(b_{2}-m \ell\right)\right)-\Upsilon(\zeta)\right]^{\frac{p \alpha}{\kappa}} d \zeta .
$$

COROLlaRY 2.13. For $\lambda=0, \forall \xi \in[0, \zeta], \varpi_{\Upsilon}(\ell, \zeta)=\zeta\left(\Upsilon\left(m b_{1}+\mathscr{F}_{\rho, \delta}^{\sigma}\left(\ell-m b_{1}\right)\right)-\right.$ $\zeta)^{\alpha-1}$ and $\forall \xi \in[\zeta, 1], \varpi_{\Upsilon}(\ell, \zeta)=\zeta\left(\Upsilon\left(m \ell+\mathscr{F}_{\rho, \delta}^{\sigma}\left(b_{2}-m \ell\right)\right)-\zeta\right)^{\alpha-1}$ in Theorem 2.3, we have

$$
\begin{aligned}
\left|T_{\Lambda, \Pi_{m}^{\mathrm{r}}, \Xi_{m}^{\Upsilon}}\left(0 ; \ell, b_{1}, b_{2}\right)\right| \leq & \frac{\left[\mathscr{F}_{\rho, \delta}^{\sigma}\left(\ell-m b_{1}\right)\right]^{\frac{q+1}{q}}}{\sqrt[q]{2}\left[\Upsilon\left(m b_{1}+\mathscr{F}_{\rho, \delta}^{\sigma}\left(\ell-m b_{1}\right)\right)-\Upsilon\left(m b_{1}\right)\right] \mathscr{F}_{\rho, \delta}^{\sigma}\left(b_{2}-m b_{1}\right)} \\
& \times \sqrt[p]{B_{7}^{\Upsilon}(\ell ; p)} \times \sqrt[q]{\left|\Lambda^{\prime}\left(m b_{1}\right)\right|^{q}+\left|\Lambda^{\prime}(\ell)\right|^{q}}
\end{aligned}
$$




$$
\begin{gathered}
+\frac{\left[\mathscr{F}_{\rho, \delta}^{\sigma}\left(b_{2}-m \ell\right)\right]^{\frac{q+1}{q}}}{\sqrt[q]{2}\left[\Upsilon^{\alpha}\left(m \ell+\mathscr{F}_{\rho, \delta}^{\sigma}\left(b_{2}-m \ell\right)\right)-\Upsilon^{\alpha}(m \ell)\right] \mathscr{F}_{\rho, \delta}^{\sigma}\left(b_{2}-m b_{1}\right)} \\
\times \sqrt[p]{B_{8}^{\Upsilon}(\ell ; p, \alpha)} \times \sqrt[q]{\left|\Lambda^{\prime}(m \ell)\right|^{q}+\left|\Lambda^{\prime}\left(b_{2}\right)\right|^{q}},
\end{gathered}
$$

where

$$
B_{7}^{\Upsilon}(\ell ; p):=\int_{m b_{1}}^{m b_{1}+\mathscr{F}_{\rho, \delta}^{\sigma}\left(\ell-m b_{1}\right)}\left[\Upsilon(\zeta)-\Upsilon\left(m b_{1}\right)\right]^{p} d \zeta
$$

and

$$
B_{8}^{\Upsilon}(\ell ; p, \alpha):=\int_{m \ell}^{m \ell+\mathscr{F}_{\rho, \delta}^{\sigma}\left(b_{2}-m \ell\right)}\left[\Upsilon^{\alpha}\left(m \ell+\mathscr{F}_{\rho, \delta}^{\sigma}\left(b_{2}-m \ell\right)\right)-\Upsilon^{\alpha}(\zeta)\right]^{p} d \zeta .
$$

COROLlaRY 2.14. Substituting $\lambda=0$ and $\varpi(\zeta)=\frac{\zeta}{\alpha} \exp (-A \zeta)$, where $A=\frac{1-\alpha}{\alpha}$, in Theorem 2.3, we get

$$
\begin{gathered}
\left|T_{\Lambda, \Pi_{m}^{\mathrm{r}}, \Xi_{m}^{\mathrm{r}}}\left(0 ; \ell, b_{1}, b_{2}\right)\right| \leq \frac{\left[\mathscr{F}_{\rho, \delta}^{\sigma}\left(\ell-m b_{1}\right)\right]^{\frac{q+1}{q}}}{\sqrt[q]{2} \mathscr{F}_{\rho, \delta}^{\sigma}\left(b_{2}-m b_{1}\right)} \\
\times \sqrt[p]{B_{9}^{\Upsilon}(\ell ; p, A)} \times \sqrt[q]{\left|\Lambda^{\prime}\left(m b_{1}\right)\right|^{q}+\left|\Lambda^{\prime}(\ell)\right|^{q}} \\
+\frac{\left[\mathscr{F}_{\rho, \delta}^{\sigma}\left(b_{2}-m \ell\right)\right]^{\frac{q+1}{q}}}{\sqrt[q]{2} \mathscr{F}_{\rho, \delta}^{\sigma}\left(b_{2}-m b_{1}\right)} \sqrt[p]{B_{10}^{\Upsilon}(\ell ; p, A)} \times \sqrt[q]{\left|\Lambda^{\prime}(m \ell)\right|^{q}+\left|\Lambda^{\prime}\left(b_{2}\right)\right|^{q}}
\end{gathered}
$$

where

$$
B_{9}^{\Upsilon}(\ell ; p, A):=\int_{m b_{1}}^{m b_{1}+\mathscr{F}_{\rho, \delta}^{\sigma}\left(\ell-m b_{1}\right)}\left\{1-\exp \left[A\left(\Upsilon\left(m b_{1}\right)-\Upsilon(\zeta)\right)\right]\right\}^{p} d \zeta
$$

and

$$
B_{10}^{\Upsilon}(\ell ; p, A):=\int_{m \ell}^{m \ell+\mathscr{F}_{\rho, \delta}^{\sigma}\left(b_{2}-m \ell\right)}\left\{1-\exp \left[A\left(\Upsilon(\zeta)-\Upsilon\left(m \ell+\mathscr{F}_{\rho, \delta}^{\sigma}\left(b_{2}-m \ell\right)\right)\right)\right]\right\}^{p} d \zeta .
$$

THEOREM 2.15. Let $\Lambda: \mathscr{P} \rightarrow \mathbb{R}$ be a differentiable function on $\mathscr{P}{ }^{\circ}$ and $\lambda \in[0,1]$. If $\left|\Lambda^{\prime}\right|^{q}$ is generalized convex on $\mathscr{P}$ and $\mathscr{F}_{\rho, \delta}^{\sigma}\left(b_{2}-m b_{1}\right)>0$, then for $q \geq 1$, we have

$$
\begin{aligned}
& \left|T_{\Lambda, \Pi_{m}^{\sigma, \mathrm{r}}, \Xi_{m}^{\sigma, \mathrm{r}}}\left(\lambda ; \ell, b_{1}, b_{2}\right)\right| \leq \frac{\left[\mathscr{F}_{\rho, \delta}^{\sigma}\left(\ell-m b_{1}\right)\right]^{2}}{\Pi_{m}^{\sigma, \mathrm{r}}(\ell, 1) \mathscr{F}_{\rho, \delta}^{\sigma}\left(b_{2}-m b_{1}\right)}\left[B_{\Pi_{m}}^{\sigma, \mathrm{r}}(\ell ; \lambda, 1)\right]^{1-\frac{1}{q}} \\
& \times \sqrt[q]{\left[B_{\Pi_{m}}^{\sigma, \Upsilon}(\ell ; \lambda, 1)-E_{\Pi_{m}}^{\sigma, \Upsilon}(\ell ; \lambda)\right]\left|\Lambda^{\prime}\left(m b_{1}\right)\right|^{q}+E_{\Pi_{m}}^{\sigma, \Upsilon}(\ell ; \lambda)\left|\Lambda^{\prime}(\ell)\right|^{q}}
\end{aligned}
$$




$$
\begin{gathered}
+\frac{\left[\mathscr{F}_{\rho, \delta}^{\sigma}\left(b_{2}-m \ell\right)\right]^{2}}{\Xi_{m}^{\sigma, \mathrm{r}}(\ell, 0) \mathscr{F}_{\rho, \delta}^{\sigma}\left(b_{2}-m b_{1}\right)}\left[B_{\Xi_{m}}^{\sigma, \mathrm{r}}(\ell ; \lambda, 1)\right]^{1-\frac{1}{q}} \\
\times \sqrt[q]{\left[B_{\Xi_{m}}^{\sigma, \mathrm{r}}(\ell ; \lambda, 1)-G_{\Xi_{m}}^{\Phi, \mathrm{r}}(\ell ; \lambda)\right]\left|\Lambda^{\prime}(m \ell)\right|^{q}+G_{\Xi_{m}}^{\sigma, \mathrm{r}}(\ell ; \lambda)\left|\Lambda^{\prime}\left(b_{2}\right)\right|^{q}},
\end{gathered}
$$

where

$$
E_{\Pi_{m}}^{\varpi, \mathrm{r}}(\ell ; \lambda):=\int_{0}^{1} \zeta\left|\Pi_{m}^{\sigma, \Upsilon}(\ell, \zeta)-\lambda\right| d \zeta, \quad G_{\Xi_{m}}^{\sigma, \Upsilon}(\ell ; \lambda):=\int_{0}^{1} \zeta\left|\Xi_{m}^{\varpi, \mathrm{r}}(\ell, \zeta)-\lambda\right| d \zeta .
$$

ProOF. By Lemma 2.1, generalized convexity of $\left|\Lambda^{\prime}\right|^{q}$ and power mean inequality, we get

$$
\begin{aligned}
& \left|T_{\Lambda, \Pi_{m}^{\sigma, \mathrm{r}}, \mathbb{\Sigma}_{m}^{\bar{\sigma}}}\left(\lambda ; \ell, b_{1}, b_{2}\right)\right| \\
& \leq \frac{\left[\mathscr{F}_{\rho, \delta}^{\sigma}\left(\ell-m b_{1}\right)\right]^{2}}{\Pi_{m}^{\sigma, \mathrm{r}}(\ell, 1) \mathscr{F}_{\rho, \delta}^{\sigma}\left(b_{2}-m b_{1}\right)} \times \int_{0}^{1}\left|\Pi_{m}^{\sigma, \mathrm{r}}(\ell, \zeta)-\lambda\right|\left|\Lambda^{\prime}\left(m b_{1}+\zeta \mathscr{F}_{\rho, \delta}^{\sigma}\left(\ell-m b_{1}\right)\right)\right| d \zeta \\
& +\frac{\left[\mathscr{F}_{\rho, \delta}^{\sigma}\left(b_{2}-m \ell\right)\right]^{2}}{\Xi_{m}^{\sigma, \mathrm{r}}(\ell, 0) \mathscr{F}_{\rho, \delta}^{\sigma}\left(b_{2}-m b_{1}\right)} \times \int_{0}^{1}\left|\Xi_{m}^{\sigma, \mathrm{r}}(\ell, \zeta)-\lambda\right|\left|\Lambda^{\prime}\left(m \ell+\zeta \mathscr{F}_{\rho, \delta}^{\sigma}\left(b_{2}-m \ell\right)\right)\right| d \zeta \\
& \leq \frac{\left[\mathscr{F}_{\rho, \delta}^{\sigma}\left(\ell-m b_{1}\right)\right]^{2}}{\prod_{m}^{\sigma, r}(\ell, 1) \mathscr{F}_{\rho, \delta}^{\sigma}\left(b_{2}-m b_{1}\right)} \\
& \times\left(\int_{0}^{1}\left|\Pi_{m}^{\Phi, \Upsilon}(\ell, \zeta)-\lambda\right| d \zeta\right)^{1-\frac{1}{q}}\left(\int_{0}^{1}\left|\Pi_{m}^{\sigma, \Upsilon}(\ell, \zeta)-\lambda \| \Lambda^{\prime}\left(m b_{1}+\zeta \mathscr{F}_{\rho, \delta}^{\sigma}\left(\ell-m b_{1}\right)\right)\right|^{q} d \zeta\right)^{\frac{1}{q}} \\
& +\frac{\left[\mathscr{F}_{\rho, \delta}^{\sigma}\left(b_{2}-m \ell\right)\right]^{2}}{\Xi_{m}^{\sigma, \mathrm{r}}(\ell, 0) \mathscr{F}_{\rho, \delta}^{\sigma}\left(b_{2}-m b_{1}\right)} \\
& \times\left(\int_{0}^{1}\left|\Xi_{m}^{\sigma, \mathrm{r}}(\ell, \zeta)-\lambda\right| d \zeta\right)^{1-\frac{1}{q}}\left(\int_{0}^{1}\left|\Xi_{m}^{\sigma, \mathrm{r}}(\ell, \zeta)-\lambda \| \Lambda^{\prime}\left(m \ell+\zeta \mathscr{F}_{\rho, \delta}^{\sigma}\left(b_{2}-m \ell\right)\right)\right|^{q} d \zeta\right)^{\frac{1}{q}} \\
& \leq \frac{\left[\mathscr{F}_{\rho, \delta}^{\sigma}\left(\ell-m b_{1}\right)\right]^{2}}{\prod_{m}^{\sigma, \mathrm{r}}(\ell, 1) \mathscr{F}_{\rho, \delta}^{\sigma}\left(b_{2}-m b_{1}\right)} \sqrt[p]{B_{\Pi_{m}}^{\sigma, \mathrm{r}}(\ell ; \lambda, p)} \\
& \times\left(\int_{0}^{1}\left|\Pi_{m}^{\Phi, r}(\ell, \zeta)-\lambda\right|\left[(1-\zeta)\left|\Lambda^{\prime}\left(m b_{1}\right)\right|^{q}+\zeta\left|\Lambda^{\prime}(\ell)\right|^{q}\right] d \zeta\right)^{\frac{1}{q}}
\end{aligned}
$$




$$
\begin{aligned}
& +\frac{\left[\mathscr{F}_{\rho, \delta}^{\sigma}\left(b_{2}-m \ell\right)\right]^{2}}{\Xi_{m}^{\sigma, \mathrm{r}}(\ell, 0) \mathscr{F}_{\rho, \delta}^{\sigma}\left(b_{2}-m b_{1}\right)} \sqrt[p]{B_{\Xi_{m}}^{\sigma, \mathrm{r}}(\ell ; \lambda, p)} \\
& \times\left(\int_{0}^{1}\left|\Xi_{m}^{\sigma, \Upsilon}(\ell, \zeta)-\lambda\right|\left[(1-\zeta)\left|\Lambda^{\prime}(m \ell)\right|^{q}+\zeta\left|\Lambda^{\prime}\left(b_{2}\right)\right|^{q}\right] d \zeta\right)^{\frac{1}{q}} \\
& =\frac{\left[\mathscr{F}_{\rho, \delta}^{\sigma}\left(\ell-m b_{1}\right)\right]^{2}}{\Pi_{m}^{\sigma, \mathrm{r}}(\ell, 1) \mathscr{F}_{\rho, \delta}^{\sigma}\left(b_{2}-m b_{1}\right)}\left[B_{\Pi_{m}}^{\sigma, \mathrm{\Upsilon}}(\ell ; \lambda, 1)\right]^{1-\frac{1}{q}} \\
& \times \sqrt[q]{\left[B_{\Pi_{m}}^{\sigma, \Upsilon}(\ell ; \lambda, 1)-E_{\Pi_{m}}^{\sigma, \Upsilon}(\ell ; \lambda)\right]\left|\Lambda^{\prime}\left(m b_{1}\right)\right|^{q}+E_{\Pi_{m}}^{\sigma, \Upsilon}(\ell ; \lambda)\left|\Lambda^{\prime}(\ell)\right|^{q}} \\
& +\frac{\left[\mathscr{F}_{\rho, \delta}^{\sigma}\left(b_{2}-m \ell\right)\right]^{2}}{\Xi_{m}^{\sigma, \Upsilon}(\ell, 0) \mathscr{F}_{\rho, \delta}^{\sigma}\left(b_{2}-m b_{1}\right)}\left[B_{\Xi_{m}}^{\sigma, \Upsilon}(\ell ; \lambda, 1)\right]^{1-\frac{1}{q}} \\
& \times \sqrt[q]{\left[B_{\Xi_{m}}^{\sigma, \mathrm{r}}(\ell ; \lambda, 1)-G_{\Xi_{m}}^{\Phi, \mathrm{r}}(\ell ; \lambda)\right]\left|\Lambda^{\prime}(m \ell)\right|^{q}+G_{\Xi_{m}}^{\Phi, \mathrm{r}}(\ell ; \lambda)\left|\Lambda^{\prime}\left(b_{2}\right)\right|^{q}} .
\end{aligned}
$$

COROllary 2.16. For $q=1$ in Theorem 2.15, we get

$$
\begin{gathered}
\left|T_{\Lambda, \Pi_{m}^{\Pi, \mathrm{r}}, \Xi_{m}^{\sigma, \mathrm{r}}}\left(\lambda ; \ell, b_{1}, b_{2}\right)\right| \leq \frac{\left[\mathscr{F}_{\rho, \delta}^{\sigma}\left(\ell-m b_{1}\right)\right]^{2}}{\Pi_{m}^{\sigma, \mathrm{r}}(\ell, 1) \mathscr{F}_{\rho, \delta}^{\sigma}\left(b_{2}-m b_{1}\right)} \\
\times\left[\left(B_{\Pi_{m}}^{\sigma, \mathrm{r}}(\ell ; \lambda, 1)-E_{\Pi_{m}}^{\sigma, \mathrm{r}}(\ell ; \lambda)\right)\left|\Lambda^{\prime}\left(m b_{1}\right)\right|+E_{\Pi_{m}}^{\sigma, \mathrm{r}}(\ell ; \lambda)\left|\Lambda^{\prime}(\ell)\right|\right] \\
+\frac{\left[\mathscr{F}_{\rho, \delta}^{\sigma}\left(b_{2}-m \ell\right)\right]^{2}}{\Xi_{m}^{\sigma, \mathrm{r}}(\ell, 0) \mathscr{F}_{\rho, \delta}^{\sigma}\left(b_{2}-m b_{1}\right)} \\
\times\left[\left(B_{\Xi_{m}}^{\sigma, \mathrm{r}}(\ell ; \lambda, 1)-G_{\Xi_{m}}^{\sigma, \mathrm{r}}(\ell ; \lambda)\right)\left|\Lambda^{\prime}(m \ell)\right|+G_{\Xi_{m}}^{\sigma, \mathrm{r}}(\ell ; \lambda)\left|\Lambda^{\prime}\left(b_{2}\right)\right|\right] .
\end{gathered}
$$

COROLlary 2.17. Taking $\left|\Lambda^{\prime}\right| \leq \mathscr{K}$ in Theorem 2.15, we have

$$
\begin{gathered}
\left|T_{\Lambda, \Pi_{m}^{\sigma, \mathrm{r}}, \Xi_{m}^{\Phi, \mathrm{r}}}\left(\lambda ; \ell, b_{1}, b_{2}\right)\right| \leq \frac{\mathscr{K}}{\mathscr{F}_{\rho, \delta}^{\sigma}\left(b_{2}-m b_{1}\right)} \\
\times\left[\frac{\left[\mathscr{F}_{\rho, \delta}^{\sigma}\left(\ell-m b_{1}\right)\right]^{2}}{\Pi_{m}^{\sigma, \mathrm{Y}}(\ell, 1)} B_{\Pi_{m}}^{\sigma, \mathrm{r}}(\ell ; \lambda, 1)+\frac{\left[\mathscr{F}_{\rho, \delta}^{\sigma}\left(b_{2}-m \ell\right)\right]^{2}}{\Xi_{m}^{\sigma, \mathrm{r}}(\ell, 0)} B_{\Xi_{m}}^{\sigma, \mathrm{r}}(\ell ; \lambda, 1)\right] .
\end{gathered}
$$


Corollary 2.18. Choosing $m=1, \lambda=0, \mathscr{F}_{\rho, \delta}^{\sigma}\left(\ell-m b_{1}\right)=\ell-m b_{1}, \mathscr{F}_{\rho, \delta}^{\sigma}\left(b_{2}-\right.$ $m \ell)=b_{2}-m \ell, \mathscr{F}_{\rho, \delta}^{\sigma}\left(b_{2}-m b_{1}\right)=b_{2}-m b_{1}$ and $\Upsilon(\zeta)=\zeta=\varpi(\zeta)$ in Theorem 2.15, we get

$$
\begin{gathered}
\left|T_{\Lambda}\left(\ell, b_{1}, b_{2}\right)\right| \leq \frac{1}{2 \sqrt[q]{3}\left(b_{2}-b_{1}\right)} \\
\times\left\{\left(\ell-b_{1}\right)^{2} \sqrt[q]{\left|\Lambda^{\prime}\left(b_{1}\right)\right|^{q}+2\left|\Lambda^{\prime}(\ell)\right|^{q}}+\left(b_{2}-\ell\right)^{2} \sqrt[q]{2\left|\Lambda^{\prime}(\ell)\right| q+\left|\Lambda^{\prime}\left(b_{2}\right)\right|^{q}}\right\} .
\end{gathered}
$$

COROLlary 2.19. Taking $\ell=\frac{b_{1}+b_{2}}{2}$ in Corollary 2.18, we obtain

$$
\begin{gathered}
\left|T_{\Lambda}\left(b_{1}, b_{2}\right)\right| \leq \frac{\left(b_{2}-b_{1}\right)}{8 \sqrt[q]{3}} \\
\times\left\{\sqrt[q]{\left|\Lambda^{\prime}\left(b_{1}\right)\right|^{q}+2\left|\Lambda^{\prime}\left(\frac{b_{1}+b_{2}}{2}\right)\right|^{q}}+\sqrt[q]{2\left|\Lambda^{\prime}\left(\frac{b_{1}+b_{2}}{2}\right)\right|^{q}+\left|\Lambda^{\prime}\left(b_{2}\right)\right|^{q}}\right\} .
\end{gathered}
$$

COROLlary 2.20. Choosing $m=1, \lambda=1, \mathscr{F}_{\rho, \delta}^{\sigma}\left(\ell-m b_{1}\right)=\ell-m b_{1}, \mathscr{F}_{\rho, \delta}^{\sigma}\left(b_{2}-\right.$ $m \ell)=b_{2}-m \ell, \underset{\rho, \delta}{\sigma}\left(b_{2}-m b_{1}\right)=b_{2}-m b_{1}$ and $\Upsilon(\zeta)=\zeta=\varpi(\zeta)$ in Theorem 2.15, we have

$$
\begin{gathered}
\left|\overline{T_{\Lambda}}\left(\ell, b_{1}, b_{2}\right)\right| \leq \frac{1}{2 \sqrt[q]{3}\left(b_{2}-b_{1}\right)} \\
\times\left\{\left(\ell-b_{1}\right)^{2} \sqrt[q]{2\left|\Lambda^{\prime}\left(b_{1}\right)\right|^{q}+\left|\Lambda^{\prime}(\ell)\right|^{q}}+\left(b_{2}-\ell\right)^{2} \sqrt[q]{\left|\Lambda^{\prime}(\ell)\right|^{q}+2\left|\Lambda^{\prime}\left(b_{2}\right)\right|^{q}}\right\} .
\end{gathered}
$$

COROLlary 2.21. Taking $m=1, \lambda=\frac{1}{3}, \mathscr{F}_{\rho, \delta}^{\sigma}\left(\ell-m b_{1}\right)=$ $\ell-m b_{1}, \mathscr{F}_{\rho, \delta}^{\sigma}\left(b_{2}-m \ell\right)=b_{2}-m \ell, \underset{\rho, \delta}{\sigma}\left(b_{2}-m b_{1}\right)=b_{2}-m b_{1}$ and $\Upsilon(\zeta)=\zeta=\varpi(\zeta)$ in Theorem 2.15, we get

$$
\begin{gathered}
\left|T_{\Lambda}\left(\frac{1}{3} ; b_{1}, b_{2}\right)\right| \leq \frac{1}{2 \sqrt[q]{243}\left(b_{2}-b_{1}\right)} \\
\times\left\{\left(\ell-b_{1}\right)^{2} \sqrt[q]{185\left|\Lambda^{\prime}\left(b_{1}\right)\right|^{q}+58\left|\Lambda^{\prime}(\ell)\right|^{q}}+\left(b_{2}-\ell\right)^{2} \sqrt[q]{195\left|\Lambda^{\prime}(\ell)\right|^{q}+48\left|\Lambda^{\prime}\left(b_{2}\right)\right|^{q}}\right\} .
\end{gathered}
$$

Corollary 2.22. Substituting $\lambda=0$ and $\varpi(\zeta)=\zeta$ in Theorem 2.15, we obtain

$$
\left|T_{\Lambda, \Pi_{m}, \Xi_{m}^{\mathrm{r}}}^{\mathrm{r}}\left(0 ; \ell, b_{1}, b_{2}\right)\right| \leq \frac{1}{\left[\mathscr{F}_{\rho, \delta}^{\sigma}\left(\ell-m b_{1}\right)\right]^{\frac{q+1}{q}} \mathscr{F}_{\rho, \delta}^{\sigma}\left(b_{2}-m b_{1}\right)}
$$




$$
\begin{gathered}
\times\left[B_{1}^{\Upsilon}(\ell ; 1)\right] \sqrt[1-\frac{1}{q}]{\sqrt[q]{\left[B_{1}^{\Upsilon}(\ell ; 1) \mathscr{F}_{\rho, \delta}^{\sigma}\left(\ell-m b_{1}\right)-C_{1}^{\Upsilon}(\ell)\right]\left|\Lambda^{\prime}\left(m b_{1}\right)\right|^{q}+C_{1}^{\Upsilon}(\ell)\left|\Lambda^{\prime}(\ell)\right|^{q}}} \\
+\frac{1}{\left[\mathscr{F}_{\rho, \delta}^{\sigma}\left(b_{2}-m \ell\right)\right]^{\frac{q+1}{q}} \mathscr{F}_{\rho, \delta}^{\sigma}\left(b_{2}-m b_{1}\right)}\left[B_{2}^{\Upsilon}(\ell ; 1)\right]^{1-\frac{1}{q}} \\
\times \sqrt[q]{\left[B_{2}^{\Upsilon}(\ell ; 1) \mathscr{F}_{\rho, \delta}^{\sigma}\left(b_{2}-m \ell\right)-E_{1}^{\Upsilon}(\ell)\right]\left|\Lambda^{\prime}(m \ell)\right|^{q}+E_{1}^{\Upsilon}(\ell)\left|\Lambda^{\prime}\left(b_{2}\right)\right|^{q}}
\end{gathered}
$$

where

$$
\begin{gathered}
C_{1}^{\Upsilon}(\ell):=\int_{m b_{1}}^{m b_{1}+\mathscr{F}_{\rho, \delta}^{\sigma}\left(\ell-m b_{1}\right)}\left(\zeta-m b_{1}\right)\left(\Upsilon(\zeta)-\Upsilon\left(m b_{1}\right)\right) d \zeta \\
E_{1}^{\Upsilon}(\ell):=\int_{m \ell}^{m \ell+\mathscr{F}_{\rho, \delta}^{\sigma}\left(^{\left(b_{2}-m \ell\right)}\right.}(\zeta-m \ell)\left(\Upsilon\left(m \ell+\mathscr{F}_{\rho, \delta}^{\sigma}\left(b_{2}-m \ell\right)\right)-\Upsilon(\zeta)\right) d \zeta .
\end{gathered}
$$

Corollary 2.23. For $\lambda=0$ and $\varpi(\zeta)=\frac{\zeta^{\alpha}}{\Gamma(\alpha)}$ in Theorem 2.15, we have

$$
\begin{gathered}
\left|T_{\Lambda, \Pi_{m}^{\Upsilon}, \Xi_{m}^{\mathrm{r}}}\left(0 ; \ell, b_{1}, b_{2}\right)\right| \leq \frac{1}{\left[\mathscr{F}_{\rho, \delta}^{\sigma}\left(\ell-m b_{1}\right)\right]^{\frac{q+1}{q}} \mathscr{F}_{\rho, \delta}^{\sigma}\left(b_{2}-m b_{1}\right)} \\
\times\left[B_{3}^{\Upsilon}(\ell ; 1, \alpha)\right]^{1-\frac{1}{q}} \\
\times \sqrt[q]{\left[B_{3}^{\Upsilon}(\ell ; 1, \alpha) \mathscr{F}_{\rho, \delta}^{\sigma}\left(\ell-m b_{1}\right)-C_{1}^{\Upsilon}(\ell, \alpha)\right]\left|\Lambda^{\prime}\left(m b_{1}\right)\right|^{q}+C_{1}^{\mathrm{r}}(\ell, \alpha)\left|\Lambda^{\prime}(\ell)\right|^{q}} \\
+\frac{1}{\left[\mathscr{F}_{\rho, \delta}^{\sigma}\left(b_{2}-m \ell\right)\right]^{\frac{q+1}{q}} \mathscr{F}_{\rho, \delta}^{\sigma}\left(b_{2}-m b_{1}\right)}\left[B_{4}^{\Upsilon}(\ell ; 1, \alpha)\right]^{1-\frac{1}{q}} \\
\times \sqrt[q]{\left[B_{4}^{\Upsilon}(\ell ; 1, \alpha) \mathscr{F}_{\rho, \delta}^{\sigma}\left(b_{2}-m \ell\right)-E_{1}^{\Upsilon}(\ell, \alpha)\right]\left|\Lambda^{\prime}(m \ell)\right|^{q}+E_{1}^{\Upsilon}(\ell, \alpha)\left|\Lambda^{\prime}\left(b_{2}\right)\right|^{q}},
\end{gathered}
$$

where

$$
\begin{gathered}
C_{1}^{\Upsilon}(\ell, \alpha):=\int_{m b_{1}}^{m b_{1}+\mathscr{F}_{\rho, \delta}^{\sigma}\left(\ell-m b_{1}\right)}\left(\zeta-m b_{1}\right)\left[\Upsilon(\zeta)-\Upsilon\left(m b_{1}\right)\right]^{\alpha} d \zeta, \\
E_{1}^{\Upsilon}(\ell, \alpha):=\int_{m \ell}^{m \ell+\mathscr{F}_{\rho, \delta}^{\sigma}\left(b_{2}-m \ell\right)}(\zeta-m \ell)\left[\Upsilon\left(m \ell+\mathscr{F}_{\rho, \delta}^{\sigma}\left(b_{2}-m \ell\right)\right)-\Upsilon(\zeta)\right]^{\alpha} d \zeta .
\end{gathered}
$$

COROLlaRY 2.24. Substituting $\lambda=0$ and $\varpi(\zeta)=\frac{\zeta^{\frac{\alpha}{\kappa}}}{\kappa \Gamma_{\kappa}(\alpha)}$ in Theorem 2.15, we 
get

$$
\begin{aligned}
& \left|T_{\Lambda, \Pi_{m}^{\mathrm{r}}, \Xi_{m}^{\mathrm{r}}}\left(0 ; \ell, b_{1}, b_{2}\right)\right| \leq \frac{1}{\left[\mathscr{F}_{\rho, \delta}^{\sigma}\left(\ell-m b_{1}\right)\right]^{\frac{q+1}{q}} \mathscr{F}_{\rho, \delta}^{\sigma}\left(b_{2}-m b_{1}\right)}\left[B_{5}^{\mathrm{\Upsilon}}(\ell ; 1, \alpha, \kappa)\right]^{1-\frac{1}{q}} \\
& \times \sqrt[q]{\left[B_{5}^{\Upsilon}(\ell ; 1, \alpha, \kappa) \mathscr{F}_{\rho, \delta}^{\sigma}\left(\ell-m b_{1}\right)-C_{1}^{\Upsilon}(\ell, \alpha, \kappa)\right]\left|\Lambda^{\prime}\left(m b_{1}\right)\right|^{q}+C_{1}^{\Upsilon}(\ell, \alpha, \kappa)\left|\Lambda^{\prime}(\ell)\right|^{q}} \\
& +\frac{1}{\left[\mathscr{F}_{\rho, \delta}^{\sigma}\left(b_{2}-m \ell\right)\right]^{\frac{q+1}{q}} \underset{\rho, \delta}{\sigma}\left(b_{2}-m b_{1}\right)}\left[B_{6}^{\Upsilon}(\ell ; 1, \alpha, \kappa)\right]^{1-\frac{1}{q}} \\
& \times \sqrt[q]{\left[B_{6}^{\mathrm{r}}(\ell ; 1, \alpha, \kappa) \mathscr{F}_{\rho, \delta}^{\sigma}\left(b_{2}-m \ell\right)-E_{1}^{\mathrm{\Upsilon}}(\ell, \alpha, \kappa)\right]\left|\Lambda^{\prime}(m \ell)\right|^{q}+E_{1}^{\mathrm{\Upsilon}}(\ell, \alpha, \kappa)\left|\Lambda^{\prime}\left(b_{2}\right)\right|^{q}},
\end{aligned}
$$

where

$$
\begin{gathered}
C_{1}^{\Upsilon}(\ell, \alpha, \kappa):=\int_{m b_{1}}^{m b_{1}+\mathscr{F}_{\rho, \delta}^{\sigma}\left(\ell-m b_{1}\right)}\left(\zeta-m b_{1}\right)\left[\Upsilon(\zeta)-\Upsilon\left(m b_{1}\right)\right]^{\frac{\alpha}{\kappa}} d \zeta, \\
E_{1}^{\Upsilon}(\ell, \alpha, \kappa):=\int_{m \ell}^{m \ell+\mathscr{F}_{\rho, \delta}^{\sigma}\left(b_{2}-m \ell\right)}(\zeta-m \ell)\left[\Upsilon\left(m \ell+\mathscr{F}_{\rho, \delta}^{\sigma}\left(b_{2}-m \ell\right)\right)-\Upsilon(\zeta)\right]^{\frac{\alpha}{\kappa}} d \zeta .
\end{gathered}
$$

COROLlaRY 2.25. For $\lambda=0, \forall \xi \in[0, \zeta], \varpi_{\Upsilon}(\ell, \zeta)=\zeta\left(\Upsilon\left(m b_{1}+\mathscr{F}_{\rho, \delta}^{\sigma}\left(\ell-m b_{1}\right)\right)-\right.$ $\zeta)^{\alpha-1}$ and $\forall \xi \in[\zeta, 1], \varpi_{\Upsilon}(\ell, \zeta)=\zeta\left(\Upsilon\left(m \ell+\mathscr{F}_{\rho, \delta}^{\sigma}\left(b_{2}-m \ell\right)\right)-\zeta\right)^{\alpha-1}$ in Theorem 2.15, we obtain

$$
\begin{gathered}
\left|T_{\Lambda, \Pi_{m}^{\Upsilon}, \Xi_{m}^{\Upsilon}}\left(0 ; \ell, b_{1}, b_{2}\right)\right| \leq \frac{1}{\left[\mathscr{F}_{\rho, \delta}^{\sigma}\left(\ell-m b_{1}\right)\right]^{\frac{q+1}{q}} \mathscr{F}_{\rho, \delta}^{\sigma}\left(b_{2}-m b_{1}\right)} \\
\times\left[B_{7}^{\Upsilon}(\ell ; 1, \alpha)\right]^{1-\frac{1}{q}} \sqrt[q]{\left[B_{7}^{\Upsilon}(\ell ; 1, \alpha) \mathscr{F}_{\rho, \delta}^{\sigma}\left(\ell-m b_{1}\right)-C_{1}^{\Upsilon}(\ell)\right]\left|\Lambda^{\prime}\left(m b_{1}\right)\right|^{q}+C_{1}^{\Upsilon}(\ell)\left|\Lambda^{\prime}(\ell)\right|^{q}} \\
+\frac{1}{\left[\mathscr{F}_{\rho, \delta}^{\sigma}\left(b_{2}-m \ell\right)\right]^{\frac{q+1}{q}} \mathscr{F}_{\rho, \delta}^{\sigma}\left(b_{2}-m b_{1}\right)}\left[B_{8}^{\Upsilon}(\ell ; 1, \alpha)\right]^{1-\frac{1}{q}} \\
\times \sqrt[q]{\left[B_{8}^{\Upsilon}(\ell ; 1, \alpha) \mathscr{F}_{\rho, \delta}^{\sigma}\left(b_{2}-m \ell\right)-L_{2}^{\Upsilon}(\ell, \alpha)\right]\left|\Lambda^{\prime}(m \ell)\right|^{q}+L_{2}^{\Upsilon}(\ell, \alpha)\left|\Lambda^{\prime}\left(b_{2}\right)\right|^{q}},
\end{gathered}
$$

where

$$
L_{2}^{\Upsilon}(\ell, \alpha):=\int_{m \ell}^{m \ell+\mathscr{F} \rho, \delta}{ }^{\left(b_{2}-m \ell\right)}(\zeta-m \ell)\left[\Upsilon^{\alpha}\left(m \ell+\mathscr{F}_{\rho, \delta}^{\sigma}\left(b_{2}-m \ell\right)\right)-\Upsilon^{\alpha}(\zeta)\right] d \zeta .
$$

Corollary 2.26. Substituting $\lambda=0$ and $\varpi(\zeta)=\frac{\zeta}{\alpha} \exp (-A \zeta)$, where $A=\frac{1-\alpha}{\alpha}$ 
in Theorem 2.15, we have

$$
\begin{gathered}
\left|T_{\Lambda, \Pi_{m}^{\Upsilon}, \Theta_{m}^{\Upsilon}}\left(0 ; \ell, b_{1}, b_{2}\right)\right| \leq \frac{1}{(1-\alpha)\left[\mathscr{F}_{\rho, \delta}^{\sigma}\left(\ell-m b_{1}\right)\right]^{\frac{q+1}{q}} \mathscr{F}_{\rho, \delta}^{\sigma}\left(b_{2}-m b_{1}\right)} \\
\times\left\{\left[B_{9}^{\Upsilon}(\ell ; 1, A)\right] \sqrt[1-\frac{1}{q}]{\sqrt[q]{L_{3}^{\Upsilon}(\ell, A)\left|\Lambda^{\prime}\left(m b_{1}\right)\right|^{q}+L_{4}^{\Upsilon}(\ell, A)\left|\Lambda^{\prime}(\ell)\right|^{q}}}\right. \\
+\frac{1}{(1-\alpha)\left[\mathscr{F}_{\rho, \delta}^{\sigma}\left(b_{2}-m \ell\right)\right]^{\frac{q+1}{q}} \mathscr{F}_{\rho, \delta}^{\sigma}\left(b_{2}-m b_{1}\right)} \\
\left.\times\left[B_{10}^{\Upsilon}(\ell ; 1, A)\right] \sqrt[1-\frac{1}{q}]{\sqrt[q]{L_{5}^{\Upsilon}(\ell, A)\left|\Lambda^{\prime}(m \ell)\right|^{q}+L_{6}^{\Upsilon}(\ell, A)\left|\Lambda^{\prime}\left(b_{2}\right)\right|^{q}}}\right\}
\end{gathered}
$$

where

$$
\begin{gathered}
L_{3}^{\Upsilon}(\ell, A):=\int_{m b_{1}}^{m b_{1}+\mathscr{F}_{\rho, \delta}^{\sigma}\left(\ell-m b_{1}\right)}\left(m b_{1}+\mathscr{F}_{\rho, \delta}^{\sigma}\left(\ell-m b_{1}\right)-\zeta\right) \\
\times\left\{1-\exp \left[A\left(\Upsilon\left(m b_{1}\right)-\Upsilon(\zeta)\right)\right]\right\} d \zeta, \\
L_{4}^{\Upsilon}(\ell, A):=\int_{m b_{1}}^{m b_{1}+\mathscr{F}_{\rho, \delta}^{\sigma}\left(\ell-m b_{1}\right)}\left(\zeta-m b_{1}\right)\left\{1-\exp \left[A\left(\Upsilon\left(m b_{1}\right)-\Upsilon(\zeta)\right)\right]\right\} d \zeta, \\
L_{5}^{\Upsilon}(\ell, A):=\int_{m \ell}^{m \ell+\mathscr{F}_{\rho, \delta}^{\sigma}\left(b_{2}-m \ell\right)}\left(m \ell+\mathscr{F}_{\rho, \delta}^{\sigma}\left(b_{2}-m \ell\right)-\zeta\right) \\
\times\left\{1-\exp \left[A\left(\Upsilon(\zeta)-\Upsilon\left(m \ell+\mathscr{F}_{\rho, \delta}^{\sigma}\left(b_{2}-m \ell\right)\right)\right)\right] d \zeta,\right. \\
L_{6}^{\Upsilon}(\ell, A):=\int_{m \ell}^{m \ell+\mathscr{F}_{\rho, \delta}^{\sigma}\left(b_{2}-m \ell\right)}(\zeta-m \ell)\left\{1-\exp \left[A\left(\Upsilon(\zeta)-\Upsilon\left(m \ell+\mathscr{F}_{\rho, \delta}^{\sigma}\left(b_{2}-m \ell\right)\right)\right)\right]\right\} d \zeta .
\end{gathered}
$$




\section{APPLICATIONS}

For $b_{1}, b_{2} \in \mathbb{R}$ and $0<b_{1}<b_{2}$ we recall:

(1) arithmetic mean:

$$
\mathrm{A}\left(b_{1}, b_{2}\right)=\frac{b_{1}+b_{2}}{2}
$$

(2) harmonic mean:

$$
\mathrm{H}\left(b_{1}, b_{2}\right)=\frac{2}{\frac{1}{b_{1}}+\frac{1}{b_{2}}} ;
$$

(3) logarithmic mean:

$$
\mathrm{L}\left(b_{1}, b_{2}\right)=\frac{b_{2}-b_{1}}{\ln \left|b_{2}\right|-\ln \left|b_{1}\right|} ;
$$

(4) generalized log-mean:

$$
\mathrm{L}_{r}\left(b_{1}, b_{2}\right)=\left[\frac{b_{2}^{r+1}-b_{1}^{r+1}}{(r+1)\left(b_{2}-b_{1}\right)}\right]^{\frac{1}{r}} ; r \in \mathbb{R} \backslash\{-1,0\} .
$$

From the main results in Section 2, we get

Proposition 3.1. Let $r, b_{1}, b_{2} \in \mathbb{R}$ with $0<b_{1}<b_{2}$, then for $r, q>1$ and $\frac{1}{p}+\frac{1}{q}=1$, we have

$$
\begin{gathered}
\left|\mathrm{A}^{r}\left(b_{1}, b_{2}\right)-\mathrm{L}_{r}^{r}\left(b_{1}, b_{2}\right)\right| \leq \frac{r\left(b_{2}-b_{1}\right)}{4 \sqrt[p]{p+1}} \\
\times\left\{\sqrt[q]{\mathrm{A}\left(b_{1}^{q(r-1)},\left(\frac{b_{1}+b_{2}}{2}\right)^{q(r-1)}\right)}+\sqrt[q]{\mathrm{A}\left(\left(\frac{b_{1}+b_{2}}{2}\right)^{q(r-1)}, b_{2}^{q(r-1)}\right)}\right\} .
\end{gathered}
$$

PROOF. Taking $m=1, \lambda=0, \ell=\frac{b_{1}+b_{2}}{2}, \mathscr{F}_{\rho, \delta}^{\sigma}\left(\ell-m b_{1}\right)=\ell-m b_{1}, \mathscr{F}_{\rho, \delta}^{\sigma}\left(b_{2}-m \ell\right)=$ $b_{2}-m \ell, \mathscr{F}_{\rho, \delta}^{\sigma}\left(b_{2}-m b_{1}\right)=b_{2}-m b_{1}, \Lambda(\zeta)=\zeta^{r}$ and $\Upsilon(\zeta)=\zeta=\varpi(\zeta)$ in Theorem 2.3, we get (69).

Proposition 3.2. Let $r, b_{1}, b_{2} \in \mathbb{R}$ with $0<b_{1}<b_{2}$, then for $r, q>1$ and $\frac{1}{p}+\frac{1}{q}=1$, we obtain

$$
\left|\mathrm{A}\left(b_{1}^{r}, b_{2}^{r}\right)-\mathrm{L}_{r}^{r}\left(b_{1}, b_{2}\right)\right| \leq \frac{r\left(b_{2}-b_{1}\right)}{4 \sqrt[p]{p+1}}
$$




$$
\times\left\{\sqrt[q]{\mathrm{A}\left(b_{1}^{q(r-1)},\left(\frac{b_{1}+b_{2}}{2}\right)^{q(r-1)}\right)}+\sqrt[q]{\mathrm{A}\left(\left(\frac{b_{1}+b_{2}}{2}\right)^{q(r-1)}, b_{2}^{q(r-1)}\right)}\right\} .
$$

PROOF. Choosing $m=1, \lambda=1, \ell=\frac{b_{1}+b_{2}}{2}, \mathscr{F}_{\rho, \delta}^{\sigma}\left(\ell-m b_{1}\right)=\ell-m b_{1}, \mathscr{F}_{\rho, \delta}^{\sigma}\left(b_{2}-\right.$ $m \ell)=b_{2}-m \ell, \mathscr{F}_{\rho, \delta}^{\sigma}\left(b_{2}-m b_{1}\right)=b_{2}-m b_{1}, \Lambda(\zeta)=\zeta^{r}$ and $\Upsilon(\zeta)=\zeta=\varpi(\zeta)$ in Theorem 2.3, we have (70).

Proposition 3.3. Let $b_{1}, b_{2} \in \mathbb{R}$ with $0<b_{1}<b_{2}$, then for $q>1$ and $\frac{1}{p}+\frac{1}{q}=1$, we get

$$
\begin{gathered}
\left|\frac{1}{\mathrm{~A}\left(b_{1}, b_{2}\right)}-\frac{1}{\mathrm{~L}\left(b_{1}, b_{2}\right)}\right| \leq \frac{\left(b_{2}-b_{1}\right)}{4 \sqrt[p]{p+1}} \\
\times\left\{\frac{1}{\sqrt[q]{\mathrm{H}\left(b_{1}^{2 q},\left(\frac{b_{1}+b_{2}}{2}\right)^{2 q}\right)}}+\frac{1}{\sqrt[q]{\mathrm{H}\left(\left(\frac{b_{1}+b_{2}}{2}\right)^{2 q}, b_{2}^{2 q}\right)}}\right\} .
\end{gathered}
$$

PROOF. Taking $m=1, \lambda=0, \ell=\frac{b_{1}+b_{2}}{2}, \mathscr{F}_{\rho, \delta}^{\sigma}\left(\ell-m b_{1}\right)=\ell-m b_{1}, \mathscr{F}_{\rho, \delta}^{\sigma}\left(b_{2}-m \ell\right)=$ $b_{2}-m \ell, \mathscr{F}_{\rho, \delta}^{\sigma}\left(b_{2}-m b_{1}\right)=b_{2}-m b_{1}, \Lambda(\zeta)=\frac{1}{\zeta}$ and $\Upsilon(\zeta)=\zeta=\varpi(\zeta)$ in Theorem 2.3, we obtain (71).

Proposition 3.4. Let $b_{1}, b_{2} \in \mathbb{R}$ with $0<b_{1}<b_{2}$, then for $q>1$ and $\frac{1}{p}+\frac{1}{q}=1$, we have

$$
\begin{gathered}
\left|\frac{1}{\mathrm{H}\left(b_{1}, b_{2}\right)}-\frac{1}{\mathrm{~L}\left(b_{1}, b_{2}\right)}\right| \leq \frac{\left(b_{2}-b_{1}\right)}{4 \sqrt[p]{p+1}} \\
\times\left\{\frac{1}{\sqrt[q]{\mathrm{H}\left(b_{1}^{2 q},\left(\frac{b_{1}+b_{2}}{2}\right)^{2 q}\right)}}+\frac{1}{\sqrt[q]{\mathrm{H}\left(\left(\frac{b_{1}+b_{2}}{2}\right)^{2 q}, b_{2}^{2 q}\right)}}\right\} .
\end{gathered}
$$

PROOF. Choosing $m=1, \lambda=1, \ell=\frac{b_{1}+b_{2}}{2}, \mathscr{F}_{\rho, \delta}^{\sigma}\left(\ell-m b_{1}\right)=\ell-m b_{1}, \mathscr{F}_{\rho, \delta}^{\sigma}\left(b_{2}-\right.$ $m \ell)=b_{2}-m \ell, \mathscr{F}_{\rho, \delta}^{\sigma}\left(b_{2}-m b_{1}\right)=b_{2}-m b_{1}, \Lambda(\zeta)=\frac{1}{\zeta}$ and $\Upsilon(\zeta)=\zeta=\varpi(\zeta)$ in Theorem 2.3, we get (72).

Proposition 3.5. Let $r, b_{1}, b_{2} \in \mathbb{R}$ with $0<b_{1}<b_{2}$ and $r>1$, then for $q \geq 1$, we obtain

$$
\left|\mathrm{A}^{r}\left(b_{1}, b_{2}\right)-\mathrm{L}_{r}^{r}\left(b_{1}, b_{2}\right)\right| \leq \sqrt[q]{\frac{2}{3}} \frac{r\left(b_{2}-b_{1}\right)}{8}
$$




$$
\times\left\{\sqrt[q]{\mathrm{A}\left(b_{1}^{q(r-1)}, 2\left(\frac{b_{1}+b_{2}}{2}\right)^{q(r-1)}\right)}+\sqrt[q]{\mathrm{A}\left(2\left(\frac{b_{1}+b_{2}}{2}\right)^{q(r-1)}, b_{2}^{q(r-1)}\right)}\right\}
$$

PROOF. Taking $m=1, \lambda=0, \ell=\frac{b_{1}+b_{2}}{2}, \mathscr{F}_{\rho, \delta}^{\sigma}\left(\ell-m b_{1}\right)=\ell-m b_{1}, \mathscr{F}_{\rho, \delta}^{\sigma}\left(b_{2}-m \ell\right)=$ $b_{2}-m \ell, \mathscr{F}_{\rho, \delta}^{\sigma}\left(b_{2}-m b_{1}\right)=b_{2}-m b_{1}, \Lambda(\zeta)=\zeta^{r}$ and $\Upsilon(\zeta)=\zeta=\varpi(\zeta)$ in Theorem 2.15, we have (73).

Proposition 3.6. Let $r, b_{1}, b_{2} \in \mathbb{R}$ with $0<b_{1}<b_{2}$ and $r>1$, then for $q \geq 1$, we get

$$
\begin{gathered}
\left|\mathrm{A}\left(b_{1}^{r}, b_{2}^{r}\right)-\mathrm{L}_{r}^{r}\left(b_{1}, b_{2}\right)\right| \leq \sqrt[q]{\frac{2}{3} \frac{r\left(b_{2}-b_{1}\right)}{8}} \\
\times\left\{\sqrt[q]{\mathrm{A}\left(2 b_{1}^{q(r-1)},\left(\frac{b_{1}+b_{2}}{2}\right)^{q(r-1)}\right)}+\sqrt[q]{\mathrm{A}\left(\left(\frac{b_{1}+b_{2}}{2}\right)^{q(r-1)}, 2 b_{2}^{q(r-1)}\right)}\right\} .
\end{gathered}
$$

PROOF. Choosing $m=1, \lambda=1, \ell=\frac{b_{1}+b_{2}}{2}, \underset{F_{, \delta}}{\sigma}\left(\ell-m b_{1}\right)=\ell-m b_{1}, \underset{F_{p, \delta}}{\sigma}\left(b_{2}-\right.$ $m \ell)=b_{2}-m \ell, \underset{\rho, \delta}{\sigma}\left(b_{2}-m b_{1}\right)=b_{2}-m b_{1}, \Lambda(\zeta)=\zeta^{r}$ and $\Upsilon(\zeta)=\zeta=\varpi(\zeta)$ in Theorem 2.15, we obtain (74).

Proposition 3.7. Let $b_{1}, b_{2} \in \mathbb{R}$ with $0<b_{1}<b_{2}$, then for $q \geq 1$, we have

$$
\begin{gathered}
\left|\frac{1}{\mathrm{~A}\left(b_{1}, b_{2}\right)}-\frac{1}{\mathrm{~L}\left(b_{1}, b_{2}\right)}\right| \leq \sqrt[q]{\frac{4}{3} \frac{\left(b_{2}-b_{1}\right)}{8}} \\
\times\left\{\frac{1}{\sqrt[q]{\mathrm{H}\left(2 b_{1}^{2 q},\left(\frac{b_{1}+b_{2}}{2}\right)^{2 q}\right)}}+\frac{1}{\sqrt[q]{\mathrm{H}\left(\left(\frac{b_{1}+b_{2}}{2}\right)^{2 q}, 2 b_{2}^{2 q}\right)}}\right\} .
\end{gathered}
$$

PROOF. Taking $m=1, \lambda=0, \ell=\frac{b_{1}+b_{2}}{2}, \mathscr{F}_{\rho, \delta}^{\sigma}\left(\ell-m b_{1}\right)=\ell-m b_{1}, \underset{\rho, \delta}{\sigma}\left(b_{2}-m \ell\right)=$ $b_{2}-m \ell, \mathscr{F}_{\rho, \delta}^{\sigma}\left(b_{2}-m b_{1}\right)=b_{2}-m b_{1}, \Lambda(\zeta)=\frac{1}{\zeta}$ and $\Upsilon(\zeta)=\zeta=\varpi(\zeta)$ in Theorem 2.15, we get (75).

PROPOSITION 3.8. Let $b_{1}, b_{2} \in \mathbb{R}$ with $0<b_{1}<b_{2}$, then for $q \geq 1$, we obtain

$$
\left|\frac{1}{\mathrm{H}\left(b_{1}, b_{2}\right)}-\frac{1}{\mathrm{~L}\left(b_{1}, b_{2}\right)}\right| \leq \sqrt[q]{\frac{4}{3}} \frac{\left(b_{2}-b_{1}\right)}{8}
$$




$$
\times\left\{\frac{1}{\sqrt[q]{\mathrm{H}\left(b_{1}^{2 q}, 2\left(\frac{b_{1}+b_{2}}{2}\right)^{2 q}\right)}}+\frac{1}{\sqrt[q]{\mathrm{H}\left(2\left(\frac{b_{1}+b_{2}}{2}\right)^{2 q}, b_{2}^{2 q}\right)}}\right\} .
$$

PROOF. Choosing $m=1, \lambda=1, \ell=\frac{b_{1}+b_{2}}{2}, \underset{F_{\rho, \delta}}{\sigma}\left(\ell-m b_{1}\right)=\ell-m b_{1}, \underset{\rho, \delta}{\sigma}\left(b_{2}-\right.$ $m \ell)=b_{2}-m \ell, \underset{\rho, \delta}{\sigma}\left(b_{2}-m b_{1}\right)=b_{2}-m b_{1}, \Lambda(\zeta)=\frac{1}{\zeta}$ and $\Upsilon(\zeta)=\zeta=\varpi(\zeta)$ in Theorem 2.15, we have (76).

Finally, we will find some new error estimations pertaining quadrature formula. Let denote $\mathscr{Q}: b_{1}=\varsigma_{0}<\varsigma_{1}<\ldots<\varsigma_{k}=b_{2}$. The following quadrature formulas are very useful in the sequel.

$$
\int_{b_{1}}^{b_{2}} \Lambda(\ell) d \ell=\mathrm{M}(\Lambda, \mathscr{Q})+\mathrm{E}(\Lambda, \mathscr{Q}), \quad \int_{b_{1}}^{b_{2}} \Lambda(\ell) d \ell=\mathrm{T}(\Lambda, \mathscr{Q})+\mathrm{E}^{*}(\Lambda, \mathscr{Q})
$$

where

$$
\mathrm{M}(\Lambda, \mathscr{Q}):=\sum_{j=0}^{k-1} \Lambda\left(\frac{\varsigma_{j}+\varsigma_{j+1}}{2}\right)\left(\varsigma_{j+1}-\varsigma_{j}\right), \quad \mathrm{T}(\Lambda, \mathscr{Q}):=\sum_{j=0}^{k-1} \frac{\Lambda\left(\varsigma_{j}\right)+\Lambda\left(\varsigma_{j+1}\right)}{2}\left(\varsigma_{j+1}-\varsigma_{j}\right),
$$

and $\mathrm{E}(\Lambda, \mathscr{Q}), \mathrm{E}^{*}(\Lambda, \mathscr{Q})$ are denoted their corresponding errors.

Proposition 3.9. Let $\Lambda:\left[b_{1}, b_{2}\right] \rightarrow \mathbb{R}$ be a differentiable function on $\left(b_{1}, b_{2}\right)$, where $b_{1}<b_{2}$. If $\left|\Lambda^{\prime}\right|^{q}$ is convex on $\left[b_{1}, b_{2}\right]$, then for $q>1$ and $\frac{1}{p}+\frac{1}{q}=1$, we have

$$
\begin{gathered}
|\mathrm{E}(\Lambda, \mathscr{Q})| \leq \frac{1}{4 \sqrt[q]{2} \sqrt[p]{p+1}} \times \sum_{j=0}^{k-1}\left(\varsigma_{j+1}-\varsigma_{j}\right)^{2} \\
\times\left\{\sqrt[q]{\left|\Lambda^{\prime}\left(\varsigma_{j}\right)\right|^{q}+\left|\Lambda^{\prime}\left(\frac{\varsigma_{j}+\varsigma_{j+1}}{2}\right)\right|^{q}}+\sqrt[q]{\left|\Lambda^{\prime}\left(\frac{\varsigma_{j}+\varsigma_{j+1}}{2}\right)\right|^{q}+\left|\Lambda^{\prime}\left(\varsigma_{j+1}\right)\right|^{q}}\right\} .
\end{gathered}
$$

ProOF. From Theorem 2.3 for $m=1, \lambda=0, \ell=\frac{b_{1}+b_{2}}{2}, \mathscr{F}_{\rho, \delta}^{\sigma}\left(\ell-m b_{1}\right)=$ $\ell-m b_{1}, \mathscr{F}_{\rho, \delta}^{\sigma}\left(b_{2}-m \ell\right)=b_{2}-m \ell, \mathscr{F}_{\rho, \delta}^{\sigma}\left(b_{2}-m b_{1}\right)=b_{2}-m b_{1}$ and $\Upsilon(\zeta)=\zeta=\varpi(\zeta)$ on $\left[\varsigma_{j}, \varsigma_{j+1}\right](j=0, \ldots, k-1)$ of $\mathscr{Q}$, we get

$$
\begin{gathered}
\left|\Lambda\left(\frac{\varsigma_{j}+\varsigma_{j+1}}{2}\right)-\frac{1}{\varsigma_{j+1}-\varsigma_{j}} \int_{\varsigma_{j}}^{\varsigma_{j+1}} \Lambda(\ell) d \ell\right| \leq \frac{\left(\varsigma_{j+1}-\varsigma_{j}\right)}{4 \sqrt[q]{2} \sqrt[p]{p+1}} \\
\times\left\{\sqrt[q]{\left|\Lambda^{\prime}\left(\varsigma_{j}\right)\right|^{q}+\left|\Lambda^{\prime}\left(\frac{\varsigma_{j}+\varsigma_{j+1}}{2}\right)\right|^{q}}+\sqrt[q]{\left|\Lambda^{\prime}\left(\frac{\varsigma_{j}+\varsigma_{j+1}}{2}\right)\right|^{q}+\left|\Lambda^{\prime}\left(\varsigma_{j+1}\right)\right|^{q}}\right\} .
\end{gathered}
$$


From (78), we have

$$
\begin{gathered}
|\mathrm{E}(\Lambda, \mathscr{Q})|=\left|\int_{b_{1}}^{b_{2}} \Lambda(\ell) d \ell-\mathrm{M}(\Lambda, \mathscr{Q})\right| \\
\leq\left|\sum_{j=0}^{k-1}\left\{\int_{\varsigma_{j}}^{\varsigma_{j+1}} \Lambda(\ell) d \ell-\Lambda\left(\frac{\varsigma_{j}+\varsigma_{j+1}}{2}\right)\left(\varsigma_{j+1}-\varsigma_{j}\right)\right\}\right| \\
\leq \sum_{j=0}^{k-1}\left|\left\{\int_{\varsigma_{j}}^{\varsigma_{j+1}} \Lambda(\ell) d \ell-\Lambda\left(\frac{\varsigma_{j}+\varsigma_{j+1}}{2}\right)\left(\varsigma_{j+1}-\varsigma_{j}\right)\right\}\right| \\
\leq\left\{\frac{1}{4 \sqrt[q]{2} \sqrt[p]{p+1}} \times \sum_{j=0}^{k-1}\left(\varsigma_{j+1}-\varsigma_{j}\right)^{2}\right. \\
\times\left\{\sqrt[q]{\left|\Lambda^{\prime}\left(\varsigma_{j}\right)\right|^{q}+\left|\Lambda^{\prime}\left(\frac{\varsigma_{j}+\varsigma_{j+1}}{2}\right)\right|^{q}}+\sqrt[q]{\left|\Lambda^{\prime}\left(\frac{\varsigma_{j}+\varsigma_{j+1}}{2}\right)\right|^{q}+\left|\Lambda^{\prime}\left(\varsigma_{j+1}\right)\right|^{q}}\right\} .
\end{gathered}
$$

Proposition 3.10. Let $\Lambda:\left[b_{1}, b_{2}\right] \rightarrow \mathbb{R}$ be a differentiable function on $\left(b_{1}, b_{2}\right)$, where $b_{1}<b_{2}$. If $\left|\Lambda^{\prime}\right|^{q}$ is convex on $\left[b_{1}, b_{2}\right]$, then for $q \geq 1$, we obtain

$$
\begin{gathered}
|\mathrm{E}(\Lambda, \mathscr{Q})| \leq \frac{1}{8 \sqrt[q]{3}} \times \sum_{j=0}^{k-1}\left(\varsigma_{j+1}-\varsigma_{j}\right)^{2} \\
\times\left\{\sqrt[q]{\left|\Lambda^{\prime}\left(\varsigma_{j}\right)\right|^{q}+2\left|\Lambda^{\prime}\left(\frac{\varsigma_{j}+\varsigma_{j+1}}{2}\right)\right|^{q}}+\sqrt[q]{2\left|\Lambda^{\prime}\left(\frac{\varsigma_{j}+\varsigma_{j+1}}{2}\right)\right|^{q}+\left|\Lambda^{\prime}\left(\varsigma_{j+1}\right)\right|^{q}}\right\} .
\end{gathered}
$$

Proof. The proof is analogous as to that of Proposition 3.9 taking $m=1, \lambda=$ $0, \ell=\frac{b_{1}+b_{2}}{2}, \mathscr{F}_{\rho, \delta}^{\sigma}\left(\ell-m b_{1}\right)=\ell-m b_{1}, \mathscr{F}_{\rho, \delta}^{\sigma}\left(b_{2}-m \ell\right)=b_{2}-m \ell, \underset{\rho, \delta}{\sigma}\left(b_{2}-m b_{1}\right)=b_{2}-m b_{1}$ and $\Upsilon(\zeta)=\zeta=\varpi(\zeta)$ using Theorem 2.15.

Proposition 3.11. Let $\Lambda:\left[b_{1}, b_{2}\right] \rightarrow \mathbb{R}$ be a differentiable function on $\left(b_{1}, b_{2}\right)$, where $b_{1}<b_{2}$. If $\left|\Lambda^{\prime}\right|^{q}$ is convex on $\left[b_{1}, b_{2}\right]$, then for $q>1$ and $\frac{1}{p}+\frac{1}{q}=1$, we have

$$
\begin{gathered}
\left|\mathrm{E}^{*}(\Lambda, \mathscr{Q})\right| \leq \frac{1}{4 \sqrt[q]{2} \sqrt[p]{p+1}} \times \sum_{j=0}^{k-1}\left(\varsigma_{j+1}-\varsigma_{j}\right)^{2} \\
\times\left\{\sqrt[q]{\left|\Lambda^{\prime}\left(\varsigma_{j}\right)\right|^{q}+\left|\Lambda^{\prime}\left(\frac{\zeta_{j}+\varsigma_{j+1}}{2}\right)\right|^{q}}+\sqrt[q]{\left|\Lambda^{\prime}\left(\frac{\zeta_{j}+\zeta_{j+1}}{2}\right)\right|^{q}+\left|\Lambda^{\prime}\left(\varsigma_{j+1}\right)\right|^{q}}\right\} .
\end{gathered}
$$


PROOF. By Theorem 2.3 for $m=1, \lambda=1, \ell=\frac{b_{1}+b_{2}}{2}, \mathscr{F}_{\rho, \delta}^{\sigma}\left(\ell-m b_{1}\right)=$ $\ell-m b_{1}, \mathscr{F}_{\rho, \delta}^{\sigma}\left(b_{2}-m \ell\right)=b_{2}-m \ell, \mathscr{F}_{\rho, \delta}^{\sigma}\left(b_{2}-m b_{1}\right)=b_{2}-m b_{1}$ and $\Upsilon(\zeta)=\zeta=\varpi(\zeta)$ on $\left[\varsigma_{j}, \varsigma_{j+1}\right](j=0, \ldots, k-1)$ of $\mathscr{Q}$, we get

$$
\begin{gathered}
\left|\frac{\Lambda\left(\varsigma_{j}\right)+\Lambda\left(\varsigma_{j+1}\right)}{2}-\frac{1}{\varsigma_{j+1}-\varsigma_{j}} \int_{\varsigma_{j}}^{\varsigma_{j+1}} \Lambda(\ell) d \ell\right| \leq \frac{\left(\varsigma_{j+1}-\varsigma_{j}\right)}{4 \sqrt[q]{2} \sqrt[p]{p+1}} \\
\times\left\{\sqrt[q]{\left|\Lambda^{\prime}\left(\varsigma_{j}\right)\right|^{q}+\left|\Lambda^{\prime}\left(\frac{\varsigma_{j}+\varsigma_{j+1}}{2}\right)\right|^{q}}+\sqrt[q]{\left|\Lambda^{\prime}\left(\frac{\varsigma_{j}+\varsigma_{j+1}}{2}\right)\right|^{q}+\left|\Lambda^{\prime}\left(\varsigma_{j+1}\right)\right|^{q}}\right\} .
\end{gathered}
$$

From (81), we get

$$
\begin{gathered}
\left|\mathrm{E}^{*}(\Lambda, \mathscr{Q})\right|=\left|\int_{b_{1}}^{b_{2}} \Lambda(\ell) d \ell-\mathrm{T}(\Lambda, \mathscr{Q})\right| \\
\leq\left|\sum_{j=0}^{k-1}\left\{\int_{\varsigma_{j}}^{\varsigma_{j+1}} \Lambda(\ell) d \ell-\frac{\Lambda\left(\varsigma_{j}\right)+\Lambda\left(\varsigma_{j+1}\right)}{2}\left(\varsigma_{j+1}-\varsigma_{j}\right)\right\}\right| \\
\leq \sum_{j=0}^{k-1}\left|\left\{\int_{\varsigma_{j}}^{\varsigma_{j+1}} \Lambda(\ell) d \ell-\frac{\Lambda\left(\varsigma_{j}\right)+\Lambda\left(\varsigma_{j+1}\right)}{2}\left(\varsigma_{j+1}-\varsigma_{j}\right)\right\}\right| \\
\times\left\{\sqrt[q]{\left|\Lambda^{\prime}\left(\varsigma_{j}\right)\right|^{q}+\left|\Lambda^{\prime}\left(\frac{\varsigma_{j}+\varsigma_{j+1}}{2}\right)\right|^{q}}+\sqrt[q]{\left|\Lambda^{\prime}\left(\frac{\varsigma_{j}+\varsigma_{j+1}}{2}\right)\right|^{q}+\left|\Lambda^{\prime}\left(\varsigma_{j+1}\right)\right|^{q}}\right\} .
\end{gathered}
$$

Proposition 3.12. Let $\Lambda:\left[b_{1}, b_{2}\right] \rightarrow \mathbb{R}$ be a differentiable function on $\left(b_{1}, b_{2}\right)$, where $b_{1}<b_{2}$. If $\left|\Lambda^{\prime}\right|^{q}$ is convex on $\left[b_{1}, b_{2}\right]$, then for $q \geq 1$, we obtain

$$
\begin{gathered}
\left|\mathrm{E}^{*}(\Lambda, Q)\right| \leq \frac{1}{8 \sqrt[q]{3}} \times \sum_{j=0}^{k-1}\left(\varsigma_{j+1}-\varsigma_{j}\right)^{2} \\
\times\left\{\sqrt[q]{2\left|\Lambda^{\prime}\left(\varsigma_{j}\right)\right|^{q}+\left|\Lambda^{\prime}\left(\frac{\zeta_{j}+\varsigma_{j+1}}{2}\right)\right|^{q}}+\sqrt[q]{\left|\Lambda^{\prime}\left(\frac{\zeta_{j}+\varsigma_{j+1}}{2}\right)\right|^{q}+2\left|\Lambda^{\prime}\left(\varsigma_{j+1}\right)\right|^{q}}\right\} .
\end{gathered}
$$

ProOF. The proof is analogous as to that of Proposition 3.11 taking $m=1, \lambda=$ $1, \ell=\frac{b_{1}+b_{2}}{2}, \mathscr{F}_{\rho, \delta}^{\sigma}\left(\ell-m b_{1}\right)=\ell-m b_{1}, \mathscr{F}_{\rho, \delta}^{\sigma}\left(b_{2}-m \ell\right)=b_{2}-m \ell, \mathscr{F}_{\rho, \delta}^{\sigma}\left(b_{2}-m b_{1}\right)=b_{2}-m b_{1}$ and $\Upsilon(\zeta)=\zeta=\varpi(\zeta)$ using Theorem 2.15. 


\section{REFERENCES}

R.P. Agarwal, M.J. Luo and R.K. Raina, On Ostrowski type inequalities. Fasc. Math. 204 (2016), 5-27.

M. Ahmadmir and R. Ullah, Some inequalities of Ostrowski and Grüss type for triple integrals on time scales. Tamkang J. Math. 42(4) (2011), 415-426.

M. Alomari, M. Darus, S.S. Dragomir and P. Cerone, Ostrowski type inequalities for functions whose derivatives are $s$-convex in the second sense. Appl. Math. Lett. 23 (2010), 1071-1076.

S.M. Aslani, M.R. Delavar and S.M. Vaezpour, Inequalities of Fejér type related to generalized convex functions with applications. Int. J. Anal. Appl. 16(1) (2018), 38-49.

F.X. Chen and S.H. Wu, Several complementary inequalities to inequalities of Hermite-Hadamard type for s-convex functions. J. Nonlinear Sci. Appl. 9(2) (2016), 705-716.

M.R. Delavar and M. De La Sen, Some generalizations of Hermite-Hadamard type inequalities. SpringerPlus 5(1661) (2016).

S.S. Dragomir, On the Ostrowski's integral inequality for mappings with bounded variation and applications. Math. Ineq. \& Appl. 1(2) (1998).

S.S. Dragomir, The Ostrowski integral inequality for Lipschitzian mappings and applications. Comput. Math. Appl. 38 (1999), 33-37.

S.S. Dragomir, Ostrowski-type inequalities for Lebesgue integral: A survey of recent results. Aust. J. Math. Anal. Appl. 14(1) (2017), 1-287.

S.S. Dragomir, Further Ostrowski and trapezoid type inequalities for the generalized Riemann-Liouville fractional integrals of functions with bounded variation. RGMIA Res. Rep. Coll. 20 (2017), Art. 84.

S.S. Dragomir, Ostrowski and trapezoid type inequalities for the generalized $k$ - $g$-fractional integrals of functions with bounded variation. RGMIA Res. Rep. Coll. 20 (2017), Art. 111.

S.S. Dragomir and R.P. Agarwal, Two inequalities for differentiable mappings and applications to special means of real numbers and trapezoidal formula. Appl. Math. Lett. 11(5) (1998), 91-95.

S.S. Dragomir and S. Wang, An inequality of Ostrowski-Grüss type and its applications to the estimation of error bounds for some special means and for some numerical quadrature rules. Comput. Math. Appl. 13(11) (1997), 15-20.

S.S. Dragomir and S. Wang, A new inequality of Ostrowski's type in $L_{1}$-norm and applications to some special means and to some numerical quadrature rules. Tamkang J. Math. 28 (1997), 239-244.

T.S. Du, M.U. Awan, A. Kashuri and S. Zhao, Some $k$-fractional extensions of the trapezium inequalities through generalized relative semi-( $m, h)$-preinvexity. Appl. Anal. (2019).

G. Farid, Existence of an integral operator and its consequences in fractional calculus. Submitted.

G. Farid, Some new Ostrowski type inequalities via fractional integrals. Int. J. Anal. Appl. 14(1) (2017), 64-68.

J. Hristov, Response functions in linear viscoelastic constitutive equations and related fractional operators. Math. Model. Nat. Phenom. 14(3) (2019), 1-34.

M. Jleli and B. Samet, On Hermite-Hadamard type inequalities via fractional integral of a function with respect to another function. J. Nonlinear Sci. Appl. 9 (2016), 1252-1260.

A. Kashuri and R. Liko, Some new Hermite-Hadamard type inequalities and their applications. Stud. Sci. Math. Hung. 56(1) (2019), 103-142. 
U.N. Katugampola, New approach to a generalized fractional integral. Appl. Math. Comput. 218(3) (2011), $860-865$.

M.A. Khan, Y.M. Chu, A. Kashuri, R. Liko and G. Ali, Conformable fractional integrals versions of Hermite-Hadamard inequalities and their generalizations. J. Funct. Spaces (2018), Article ID 6928130, pp. 9.

A.A. Kilbas, O.I. Marichev and S.G. Samko, Fractional Integrals and Derivatives. Theory and Applications. Gordon and Breach, Switzerland, (1993).

A.A. Kilbas, H.M. Srivastava, and J.J. Trujillo, Theory and Applications of Fractional Differential Equations. Elsevier Science B.V., Amsterdam, (2006).

R. Khalil, M.A. Horani, A. Yousef and M. Sababheh, A new definition of fractional derivatives. J. Comput. Appli. Math. 264 (2014), 65-70.

M. Kirane and B.T. Torebek, Hermite-Hadamard, Hermite-Hadamard-Fejér, Dragomir-Agarwal and Pachpatti type inequalities for convex functions via fractional integrals. arXive: 1701.00092.

W.J. Liu, Some Simpson type inequalities for $h$-convex and $(\alpha, m)$-convex functions. J. Comput. Anal. Appl. 16(5) (2014), 1005-1012.

W., Liu, W. Wen and J. Park, Hermite-Hadamard type inequalities for $M T$-convex functions via classical integrals and fractional integrals. J. Nonlinear Sci. Appl. 9 (2016), 766-777.

W. Liu, W. Wen and J. Park, Ostrowski type fractional integral inequalities for $M T$-convex functions. Miskolc Math. Notes 16(1) (2015), 249-256.

Z. Liu, Some Ostrowski-Grüss type inequalities and applications. Comput. Math. Appl. 53 (2007), 73-79.

Z. Liu, Some companions of an Ostrowski type inequality and applications. J. Inequal. in Pure and Appl. Math. 10(2) (2009), Art. 52, pp. 12.

M. Matloka, Ostrowski type inequalities for functions whose derivatives are $h$-convex via fractional integrals. Journal of Scientific Research and Reports 3(12) (2014), 1633-1641.

M.V. Mihai, Some Hermite-Hadamard type inequalities via Riemann-Liouville fractional calculus. Tamkang J. Math. 44(4) (2013), 411-416.

S. Mubeen and G.M. Habibullah, k-Fractional integrals and applications. Int. J. Contemp. Math. Sci. 7 (2012), 89-94.

M.A. Noor, Some new classes of nonconvex functions. Nonlinear Funct. Anal. Appl. 11 (2006), 165-171.

M.A. Noor, Some properties of nonconvex functions. Nonlinear Funct. Anal. Appl. 23(3) (2018), 575-583.

O. Omotoyinbo and A. Mogbodemu, Some new Hermite-Hadamard integral inequalities for convex functions. Int. J. Sci. Innovation Tech. 1(1) (2014), 1-12.

M.E. Özdemir, S.S. Dragomir and C. Yildiz, The Hadamard's inequality for convex function via fractional integrals. Acta Math. Sci., Ser. B, Engl. Ed. 33(5) (2013), 153-164.

M.E. Özdemir, H. Kavurmac and E. Set, Ostrowski's type inequalities for $(\alpha, m)$-convex functions. Kyungpook Math. J. 50 (2010), 371-378.

B.G. Pachpatte, On an inequality of Ostrowski type in three independent variables. J. Math. Anal. Appl. 249 (2000), 583-591.

B.G. Pachpatte, On a new Ostrowski type inequality in two independent variables. Tamkang J. Math. 32(1) (2001), 45-49. 
F. Qi and B.Y. Xi, Some integral inequalities of Simpson type for GA-E-convex functions. Georgian Math. J. 20(5) (2013), 775-788.

A. Rafiq, N. A. Mir and F. Ahmad, Weighted Čebyšev-Ostrowski type inequalities. Applied Math. Mechanics (English Ed.) 28(7) (2007), 901-906.

M.Z. Sarikaya, On the Ostrowski type integral inequality. Acta Math. Univ. Comenianae 79(1) (2010), 129-134.

M.Z. Sarikaya and F. Ertuğral, On the generalized Hermite-Hadamard inequalities. https://www . researchgate.net/publication/321760443.

M.Z. Sarikaya and H. Yildirim, On generalization of the Riesz potential. Indian Jour. of Math. and Mathematical Sci. 3(2), (2007), 231-235.

M.A. Noor, M.U. Awan and A. Gözpinar, Generalized Hermite-Hadamard type inequalities involving fractional integral operators. J. Inequal. Appl. 169 (2017), 1-10.

R.K. Raina, On generalized Wright's hypergeometric functions and fractional calculus operators. EAMJ, East Asian Math. J. 21(2) (2005), 191-203.

M. Tomar, P. Agarwal, M. Jleli and B. Samet, Certain Ostrowski type inequalities for generalized $s-$ convex functions. J. Nonlinear Sci. Appl. 10 (2017), 5947-5957.

M. Tunç, Ostrowski type inequalities for functions whose derivatives are MT-convex. J. Comput. Anal. Appl. 17(4) (2014), 691-696.

N. Ujević, Sharp inequalities of Simpson type and Ostrowski type. Comput. Math. Appl. 48 (2004), 145151.

H. Wang, T.S. Du and Y. Zhang, $k$-fractional integral trapezium-like inequalities through $(h, m)-$ convex and ( $\alpha, m)$-convex mappings. J. Inequal. Appl. 2017(311) (2017), pp. 20.

B.Y. Xi and F. Qi, Some integral inequalities of Hermite-Hadamard type for convex functions with applications to means. J. Funct. Spaces Appl. 2012 (2012), Article ID 980438, pp. 14.

Ç. Yildiz, M.E. Özdemir and M.Z. Sarikaya, New generalizations of Ostrowski-like type inequalities for fractional integrals. Kyungpook Math. J. 56 (2016), 161-172.

X.M. Zhang, Y.M. Chu and X.H. Zhang, The Hermite-Hadamard type inequality of $G A$-convex functions and its applications. J. Inequal. Appl. (2010), Article ID 507560, pp. 11.

Y. Zhang, T.S. Du, H. Wang, Y.J. Shen and A. Kashuri, Extensions of different type parameterized inequalities for generalized $(m, h)$-preinvex mappings via $k$-fractional integrals. J. Inequal. Appl. 2018 (49) (2018), pp. 30.

L. Zhongxue, On sharp inequalities of Simpson type and Ostrowski type in two independent variables. Comput. Math. Appl. 56 (2008), 2043-2047. 
Artion Kashuri

Department of Mathematics,

Faculty of Technical Science,

University “Ismail Qemali", 9400, Vlora, Albania.

email: artionkashuri@gmail.com

Muhammad Aamir Ali

Jiangsu Key Laboratory of NSLSCS,

School of Mathematical Sciences,

Nanjing Normal University, 210023, China.

email: mahr.muhammad.aamir@gmail.com

Mujahid Abbas

Department of Mathematics,

Government College University,

Lahore 54000, Pakistan

and

Department of Mathematics and Applied,

Mathematics, University of Pretoria,

Lynnwood road, Pretoria 0002, South Africa

email: abbas.mujahid@gmail.com

Muhammad Toseef

Department of Mathematics,

Government College University Lahore, Pakistan

email: toseefrana95@gmail.com 